\title{
Computing toric degenerations of flag varieties
}

Lara Bossinger, Sara Lamboglia, Kalina Mincheva, and Fatemeh Mohammadi

\begin{abstract}
We compute toric degenerations arising from the tropicalization of the full flag varieties $\mathscr{F} \ell_{4}$ and $\mathscr{F} \ell_{5}$ embedded in a product of Grassmannians. For $\mathscr{F} \ell_{4}$ and $\mathscr{F} \ell_{5}$ we compare toric degenerations arising from string polytopes and the FFLV polytope with those obtained from the tropicalization of the flag varieties. We also present a general procedure to find toric degenerations in the cases where the initial ideal arising from a cone of the tropicalization of a variety is not prime.
\end{abstract}

\section{Introduction}

Consider the variety $\mathscr{F} \ell_{n}$ of full flags $\{0\}=V_{0} \subset V_{1} \subset \cdots \subset V_{n-1} \subset V_{n}=\mathbb{C}^{n}$ of vector subspaces of $\mathbb{C}^{n}$ with $\operatorname{dim}_{\mathbb{C}}\left(V_{i}\right)=i$. The flag variety $\mathscr{F} \ell_{n}$ is naturally embedded in a product of Grassmannians using the Plücker coordinates. We denote by $I_{n}$ the defining ideal of $\mathscr{F} \ell_{n}$ with respect to this embedding. We produce toric degenerations of $\mathscr{F} \ell_{n}$ as Gröbner degenerations coming from the initial ideals associated to the maximal cones of $\operatorname{trop}\left(\mathscr{F} \ell_{n}\right)$. Moreover, we compare these with certain toric degenerations arising from representation theory.

Lara Bossinger

University of Cologne, Mathematisches Institut, Weyertal 86 - 90, 50931 Cologne, Germany.

e-mail: lbossingemath.uni-koeln.de

Sara Lamboglia

University of Warwick, Mathematics Institute, Coventry, CV4 7AL, United Kingdom.

e-mail: S. Lamboglia@warwick.ac.uk

Kalina Mincheva

Yale University, Department of Mathematics, 10 Hillhouse Ave., New Haven, CT 06511, USA.

e-mail: kalina.mincheva@yale.edu

Fatemeh Mohammadi

University of Bristol, School of Mathematics, Bristol, BS8 1TW, United Kingdom.

e-mail: fatemeh.mohammadiebristol.ac.uk 
We will consider 1-parameter toric degenerations of $\mathscr{F} \ell_{n}$. These are flat families $\varphi: \mathscr{F} \rightarrow \mathbb{A}^{1}$, where the fiber over zero (also called special fiber) is a toric variety and all other fibers are isomorphic to $\mathscr{F} \ell_{n}$. Once we have such a degeneration, some of the algebraic invariants of $\mathscr{F} \ell_{n}$ will be the same for all fibers, hence the computation can be done on the toric fiber. In the case of a toric variety such invariants are easier to compute than in the case of a general variety. In fact, they have a nice combinatorial description. Moreover, toric degenerations connect different areas of mathematics, such as symplectic geometry, representation theory, and algebraic geometry.

Let $X=V(I)$ be a projective variety and $\operatorname{trop}(X)$ be its tropicalization. The initial ideals associated to the top-dimensional cones of $\operatorname{trop}(X)$ are good candidates to give toric degenerations, see Lemma[1] (and [27, Proposition 1.1] for a more general statement). For example, in the case of $\operatorname{Grassmannians} \operatorname{Gr}\left(2, \mathbb{C}^{n}\right)$ each maximal cone of trop $\left(\operatorname{Gr}\left(2, \mathbb{C}^{n}\right)\right)$ gives a toric degeneration, see [29, 32, 5]. However, this is not true for the Grassmannians $\operatorname{Gr}\left(3, \mathbb{C}^{n}\right)$. In [27] Mohammadi and Shaw identify which maximal cones of trop $\left(\operatorname{Gr}\left(3, \mathbb{C}^{n}\right)\right)$ produce such degenerations.

The following are our main results. More detailed formulations can be found in Theorem 4, Theorem 5, and Proposition5 We will call a maximal cone $C$ of $\operatorname{trop}(X)$ prime if $\operatorname{in}_{C}(I):=\mathrm{in}_{\mathbf{w}}(I)$ is prime, with $\mathbf{w}$ a vector in the relative interior of $C$.

Theorem 1. The tropical variety $\operatorname{trop}\left(\mathscr{F} \ell_{4}\right) \subset \mathbb{R}^{14} / \mathbb{R}^{3}$ is a 6-dimensional fan with 78 maximal cones. From prime cones we obtain four non-isomorphic toric degenerations. After applying Procedure 1 we obtain at least two additional non-isomorphic toric degenerations from non-prime cones.

Theorem 2. The tropical variety trop $\left(\mathscr{F} \ell_{5}\right) \subset \mathbb{R}^{30} / \mathbb{R}^{4}$ is a 10-dimensional fan with 69780 maximal cones. From prime cones we obtain 180 non-isomorphic toric degenerations.

Toric degenerations of flag varieties and Schubert varieties have been studied intensively in representation theory over the last two decades. We refer the reader to [13] for a nice overview on this topic and to the references therein.

The main motivation of this paper is to study the flat degenerations of flag varieties into toric varieties arising from the tropicalization and to compare these degenerations to those associated to string polytopes and the Feigin-Fourier-LittelmannVinberg polytope (FFLV polytope).

Theorem 3. For $\mathscr{F} \ell_{4}$ there is at least one new toric degeneration arising from prime cones of trop $\left(\mathscr{F} \ell_{4}\right)$ in comparison to those obtained from string polytopes and the FFLV polytope.

For $\mathscr{F} \ell_{5}$ there are at least 168 new toric degenerations arising from prime cones of trop $\left(\mathscr{F} \ell_{5}\right)$ in comparison to those obtained from string polytopes and the FFLV polytope.

Our work is closely related to the theory of Newton-Okounkov bodies. Let $\mathbb{k}$ be a not necessarily algebraically closed field and $X$ a projective variety. It is possible to 
associate to every prime cone in $\operatorname{trop}(X)$ a valuation with a finite Khovanskii basis $B$ on the homogeneous coordinate ring $\mathbb{k}[X]$, see [23, Lemma 5.7]. This is a set of elements of $\mathbb{k}[X]$, such that their valuations generate the value semigroup $S(\mathbb{k}[X], \mathfrak{v})$. The convex hull of $S(\mathbb{k}[X], \mathfrak{v}) \cup\{0\}$ is referred to as the Newton-Okounkov cone. After intersecting this cone with a particular hyperplane one obtains a convex body, called the Newton-Okounkov body. When a finite Khovanskii basis exists, [2, Theorem 1.1] states that there is a flat degeneration of the variety $X$ into a toric variety whose normalization has as associated polytope the Newton-Okounkov body. In this case the Newton-Okounkov body is a polytope. The toric polytopes obtained in Theorem 4 Theorem 5, and Proposition 5 5 can be seen as Newton-Okounkov bodies for the valuations defined in $\S 6$.

The paper is structured as follows. In $\sqrt{2}$ we provide the necessary background. We study the tropicalization of the flag varieties $\mathscr{F} \ell_{n}$ for $n=4,5$ and the induced toric degenerations in 43 . The solutions to [30, Problem 5 on Grassmannians] and [30, Problem 6 on Grassmannians] can be found in Theorem 4]

In $\$ 4$ we recall the definition of string cones, string polytopes, and FFLV polytope for regular dominant integral weights. We compute for $\mathscr{F} \ell_{4}$ and $\mathscr{F} l_{5}$ all string polytopes for the weight $\rho$, which is the sum of all fundamental weights. Moreover, in $\$ 5$ for every string cone we construct a weight vector $\mathbf{w}_{\underline{w}_{0}}$ contained in the tropicalization of the flag variety in order to further explore the connection between these two different approaches. The construction is inspired by Caldero [7].

In $₫ 6$ we give an algorithmic approach to solving [23, Problem 1] for a subvariety $X$ of a toric variety $Y$ when each cone in $\operatorname{trop}(X)$ has multiplicity one. Procedure 1 aims at computing a new embedding $X^{\prime}$ of $X$ in case trop $(X)$ has some non-prime cones. Once we have such an embedding, we explain how to get new toric degenerations of $X$. We apply the procedure to $\mathscr{F} \ell_{4}$. Furthermore, we explain how to interpret the procedure in terms of finding valuations with finite Khovanskii basis on the algebra given by the homogeneous coordinate ring of $X$.

\section{Preliminary notions}

In this section we recall the definition of flag variety and we introduce the necessary background in tropical geometry. In fact, the key ingredient in the study of Gröbner toric degenerations of $\mathscr{F} \ell_{n}$ is the subfan of the Gröbner fan of $I_{n}$ given by the tropicalization of $\mathscr{F} \ell_{n}$.

We mostly refer to the approach described in [25] and we encourage the reader to look there for a more thorough introduction.

Let $\mathbb{k}$ be a field with char $(\mathbb{k})=0$ and consider on it the trivial valuation. We are mainly interested in the case when $\mathbb{k}=\mathbb{C}$.

Definition 1. A complete flag in the vector space $\mathbb{k}^{n}$ is a chain

$$
\mathscr{V}:\{0\}=V_{0} \subset V_{1} \subset \cdots \subset V_{n-1} \subset V_{n}=\mathbb{k}^{n}
$$


of vector subspaces of $\mathbb{k}^{n}$ with $\operatorname{dim}_{\mathbb{k}}\left(V_{i}\right)=i$.

The set of all complete flags in $\mathbb{k}^{n}$ is denoted by $\mathscr{F} \ell_{n}$ and it has an algebraic variety structure. More precisely, it is a subvariety of the product of Grassmannians $\operatorname{Gr}\left(1, \mathbb{k}^{n}\right) \times \operatorname{Gr}\left(2, \mathbb{k}^{n}\right) \times \cdots \times \operatorname{Gr}\left(n-1, \mathbb{k}^{n}\right)$.

Composing with the Plücker embeddings of the Grassmannians, $\mathscr{F} \ell_{n}$ becomes a subvariety of $\mathbb{P}^{\left(\begin{array}{c}n \\ 1\end{array}\right)-1} \times \mathbb{P}^{\left(\begin{array}{c}n \\ 2\end{array}\right)-1} \times \cdots \times \mathbb{P}^{\left(\begin{array}{c}n \\ n-1\end{array}\right)-1}$ and so we can ask for its defining ideal $I_{n}$. Each point in the flag variety can be represented by an $(n-1) \times n$-matrix $M=\left(x_{i, j}\right)$ whose first $d$ rows generate $V_{d}$. Each $V_{d}$ corresponds to a point in a Grassmannian. Moreover, they satisfy the condition $V_{d} \subset V_{d+1}$ for $d=0, \ldots, n-1$. In order to compute the ideal $I_{n}$ defining $\mathscr{F} \ell_{n}$ in $\mathbb{P}^{\left(\begin{array}{l}n \\ 1\end{array}\right)-1} \times \mathbb{P}^{\left(\begin{array}{c}n \\ 2\end{array}\right)-1} \times \cdots \times \mathbb{P}^{\left(\begin{array}{c}n \\ n-1\end{array}\right)-1}$ we have to translate the inclusions $V_{d} \subset V_{d+1}$ into polynomial equations. We define the map

$$
\varphi_{n}: \mathbb{k}\left[p_{J}: \varnothing \neq J \subsetneq\{1, \ldots, n\}\right] \rightarrow \mathbb{k}\left[x_{i, j}: 1 \leq i \leq n-1,1 \leq j \leq n\right]
$$

sending each Plücker variable $p_{J}$ to the determinant of the submatrix of $M$ with row indices $1, \ldots,|J|$ and column indices in $J$. The ideal $I_{n}$ of $\mathscr{F} \ell_{n}$ is the kernel of $\varphi_{n}$. There is an action of $S_{n} \rtimes \mathbb{Z}_{2}$ on $\mathscr{F} \ell_{n}$. The symmetric group acts by permuting the columns of $M$. The action of $\mathbb{Z}_{2}$ maps a complete flag $\mathscr{V}$ to its complement, which is defined to be

$$
\mathscr{V}^{\perp}:\{0\}=V_{n}^{\perp} \subset V_{n-1}^{\perp} \subset \cdots \subset V_{1}^{\perp} \subset V_{0}^{\perp}=\mathbb{k}^{n} .
$$

We will hence do computations up to $S_{n} \rtimes \mathbb{Z}_{2}$-symmetry. We are interested in finding toric degenerations. These are degenerations whose special fiber is defined by a toric ideal, i.e. a binomial prime ideal not containing monomials. This toric ideal arises as initial ideal of $I_{n}$.

Definition 2. Let $f=\sum a_{\mathbf{u}} x^{\mathbf{u}}$ with $\mathbf{u} \in \mathbb{Z}_{\geq 0}^{n+1}$ be a polynomial in $S=\mathbb{k}\left[x_{0}, \ldots, x_{n}\right]$. For each $\mathbf{w} \in \mathbb{R}^{n+1}$ we define its initial form to be

$$
\operatorname{in}_{\mathbf{w}}(f)=\sum_{\mathbf{w} \cdot \mathbf{u} \text { is minimal }} a_{\mathbf{u}} x^{\mathbf{u}} .
$$

If $I$ is an ideal in $S$, then its initial ideal with respect to $\mathbf{w}$ is

$$
\operatorname{in}_{\mathbf{w}}(I)=\left\langle\operatorname{in}_{\mathbf{w}}(f): f \in I\right\rangle .
$$

An important geometric property of initial ideals is that there exists a flat family over $\mathbb{A}^{1}$ for which the fiber over 0 is isomorphic to $V\left(\mathrm{in}_{\mathrm{w}}(I)\right)$ and all the other fibers are isomorphic to the variety $V(I)$. Here, if $J$ is a homogeneous ideal of $S$ then we define $V(J):=\operatorname{Proj}(S / J)$ where the grading on $S$ and hence on $S / J$ comes from the ambient space which has $S$ as homogeneous coordinate ring.

Let $t$ be the coordinate in $\mathbb{A}^{1}$, then the flat family is given by the ideal

$$
\tilde{I}_{t}=\left\langle t^{-\min _{\mathbf{u}}\{\mathbf{w} \cdot \mathbf{u}\}} f\left(t^{w_{0}} x_{0}, \ldots, t^{w_{n}} x_{n}\right): f=\sum a_{\mathbf{u}} x^{\mathbf{u}} \text { in } I\right\rangle \subset \mathbb{k}\left[t, x_{0}, \ldots, x_{n}\right] .
$$


This family gives a flat degeneration of the variety $V(I)$ into the variety $V\left(\operatorname{in}_{\mathbf{w}}(I)\right)$ called the Gröbner degeneration. In order to look for toric degenerations, we study the tropicalization of $V(I)$.

Definition 3. Let $f=\sum a_{\mathbf{u}} x^{\mathbf{u}}$ be any polynomial in $S$. The tropicalization of $f$ is the function $\operatorname{trop}(f): \mathbb{R}^{n+1} \rightarrow \mathbb{R}$ given by

$$
\operatorname{trop}(f)(\mathbf{w})=\min \left\{\mathbf{w} \cdot \mathbf{u}: \mathbf{u} \in \mathbb{Z}_{\geq 0}^{n+1} \text { and } a_{\mathbf{u}} \neq 0\right\} .
$$

Let $f=\sum a_{\mathbf{u}} x^{\mathbf{u}}$ be a homogeneous polynomial in $S$. Then if $\mathbf{w}-\mathbf{v}=m \cdot \mathbf{1}$, for some $\mathbf{v}, \mathbf{w} \in \mathbb{R}^{n+1}, \mathbf{1}=(1, \ldots, 1) \in \mathbb{R}^{n+1}$ and $m \in \mathbb{R}$, we have that the minimum in $\operatorname{trop}(f)(\mathbf{w})$ and $\operatorname{trop}(f)(\mathbf{v})$ is achieved for the same $\mathbf{u} \in \mathbb{Z}_{\geq 0}^{n+1}$ such that $a_{\mathbf{u}} \neq 0$.

Definition 4. Let $f$ be a homogeneous polynomial in $S$ and $V(f)$ the associated hypersurface in $\mathbb{P}^{n}$. Then the tropical hypersurface of $f$ is defined to be

$$
\operatorname{trop}(V(f))=\left\{\mathbf{w} \in \mathbb{R}^{n+1} / \mathbb{R} \mathbf{1} \cong \mathbb{R}^{n}: \begin{array}{c}
\text { the minimum in trop }(f)(\mathbf{w}) \\
\text { is achieved at least twice }
\end{array}\right\} .
$$

Let $I$ be a homogeneous ideal in $S$. The tropicalization of the variety $V(I) \subset \mathbb{P}^{n}$ is defined to be

$$
\operatorname{trop}(V(I))=\bigcap_{f \in I} \operatorname{trop}(V(f))
$$

For every $\mathbf{w} \in \operatorname{trop}(V(I)), \operatorname{in}_{\mathbf{w}}(I)$ does not contain any monomial (see proof of [25, Theorem 3.2.3]). If $V(I)$ is a $(d-1)$-dimensional irreducible projective variety, then $\operatorname{trop}(V(I))$ is the support of a rational fan given by the quotient by $\mathbb{R} \mathbf{1}$ of a subfan $F$ of the Gröbner fan of $I$ ([25, Theorem 3.3.5]). The fan $F$ has dimension $d$, which is the Krull dimension of $S / I$. It is possible to quotient by $\mathbb{R} \mathbf{1}$ because $I$ is homogeneous and hence $\operatorname{in}_{\mathbf{w}}(I)=\operatorname{in}_{\mathbf{v}}(I)$ for every $\mathbf{w}-\mathbf{v}=m \cdot \mathbf{1}$ with $\mathbf{v}, \mathbf{w} \in \mathbb{R}^{n+1}$ and $m \in \mathbb{R}$. If we consider this fan structure on $\operatorname{trop}(V(I))$ we have that vectors in the relative interior of a cone give rise to the same initial ideal and vectors in distinct relative cone interiors induce distinct initial ideals. For this reason we denote by $\operatorname{in}_{C}(I)$ the initial ideal of $I$ with respect to any $\mathbf{w}$ in the relative interior of $C$.

The tropicalization of a variety $X$ is non-empty only if $X$ intersects the torus $T^{n}=\left(\mathbb{k}^{*}\right)^{n+1} / \mathbb{k}^{*}$ non-trivially. In fact, $\operatorname{trop}(X)$ is technically the tropicalization of $X \cap T^{n}$.

In the same way the tropicalization can be defined when $S$ is the total coordinate ring (see [9, page 207] for a definition) of $\mathbb{P}^{k_{1}} \times \cdots \times \mathbb{P}^{k_{s}}$. The ring $S$ has a $\mathbb{Z}^{s}$-grading given by deg: $\mathbb{Z}^{n+1} \rightarrow \mathbb{Z}^{s}$. An ideal $I$ defining an irreducible subvariety $V(I)$ of $\mathbb{P}^{k_{1}} \times$ $\cdots \times \mathbb{P}^{k_{s}}$ is homogeneous with respect to this grading. The tropicalization of $V(I)$ is contained in $\mathbb{R}^{k_{1}+\ldots+k_{s}+s} / H$, where $H$ is an $s$-dimensional linear space spanned by the rows of the matrix $D$ associated to deg. Similarly to the projective case, if $V(I)$ is a $d$-dimensional irreducible subvariety of $\mathbb{P}^{k_{1}} \times \cdots \times \mathbb{P}^{k_{s}}$, then trop $(V(I))$ is the support of a fan, which is the quotient by $H$ of a rational $(d+s)$-dimensional subfan $F$ of the Gröbner fan of $I$. Here the Krull dimension of $S / I$ is $d+s$. 
In the following we will always consider $\operatorname{trop}(V(I))$ with the fan structure defined above.

Remark 1. A detailed definition of the tropicalization of a general toric variety $X_{\Sigma}$ and of its subvarieties can be found in [25, Chapter 6]. Note that we only consider the tropicalization of the intersection of $V(I)$ with the torus of $X_{\Sigma}$ while in [25, Chapter 6] they introduce a generalized version of $\operatorname{trop}(V(I))$ which includes the tropicalization of the intersection of $V(I)$ with each orbit of $X_{\Sigma}$.

Another property of trop $(V(I))$ is that any fan structure on it can be balanced assigning a positive integer weight to every maximal cell. We will not explain the notion of balancing in detail and we consider an adapted version of the multiplicity defined in [25, Definition 3.4.3].

Definition 5. Let $I \subset S$ be a homogeneous ideal and $\Sigma$ be a fan with support $|\Sigma|=$ $|\operatorname{trop}(V(I))|$ such that for every cone $C$ of $\Sigma$ the ideal $\operatorname{in}_{\mathbf{w}}(I)$ is constant for $\mathbf{w}$ in the relative interior of $C$. For a maximal dimensional cone $C \in \Sigma$ we define the multiplicity as mult $(C)=\sum_{P} \operatorname{mult}\left(P, \operatorname{in}_{C}(I)\right)$. Here the sum is taken over the minimal associated primes $P$ of $\operatorname{in}_{C}(I)$ that do not contain monomials (see [11, $\left.\$ 3\right]$ or [8, $\S 4.7])$.

As we have seen, each cone of trop $(V(I))$ corresponds to an initial ideal which contains no monomials. Moreover, we will see that the good candidates for toric degenerations are the initial ideals corresponding to the relative interior of the maximal cones. We say a maximal cone is prime if the corresponding initial ideal is prime.

Lemma 1. Let $I \subset S$ be a homogeneous ideal and $C$ a maximal cone of trop $(V(I))$. If $\mathrm{in}_{C}(I)$ is a toric ideal, i.e. binomial and prime, then $C$ has multiplicity one. If $C$ has multiplicity one, then $\mathrm{in}_{C}(I)$ has a unique toric ideal in its primary decomposition.

Proof. We first prove the lemma for $S$ the homogeneous coordinate ring of $\mathbb{P}^{n}$. Let $I^{\prime}=\operatorname{in}_{C}(I) \mathbb{k}\left[x_{1}^{ \pm 1}, \ldots, x_{n}^{ \pm 1}\right]$ and consider the subvariety $V\left(I^{\prime}\right)$ of the torus $T^{n}$. Then by [25, Remark 3.4.4] the multiplicity of a maximal cone $C$ is counting the number of $d$-dimensional torus orbits whose union is $V\left(I^{\prime}\right)$. If $\operatorname{in}_{C}(I)$ is toric, then $V\left(I^{\prime}\right)$ is an irreducible toric variety having a unique $d$-dimensional torus orbit. Hence $C$ has multiplicity one.

Suppose now $C$ has multiplicity one. This implies that $\operatorname{in}_{C}(I)$ contains one associated prime $J$, which does not contain monomials. The ideal $J$ has to be binomial since it is the ideal of the unique $d$-dimensional torus orbit contained in $V\left(I^{\prime}\right)$.

When $S$ is the total coordinate ring of $\mathbb{P}^{k_{1}} \times \cdots \times \mathbb{P}^{k_{s}}$, the torus is given by $T^{k_{1}} \times$ $\cdots \times T^{k_{s}} \cong T^{k_{1}+\cdots+k_{s}}$. We may assume that for each $i$,

$$
T^{k_{i}}=\left\{\left[1: a_{1}: \ldots: a_{k_{i}}\right] \in \mathbb{P}^{k_{i}}: a_{j} \neq 0 \text { for all } j\right\} .
$$

The variables for $\mathbb{P}^{k_{i}}$ are denoted by $x_{i, 0}, \ldots, x_{i, k_{i}}$ for each $i$. We fix the Laurent polynomial ring 


$$
S^{\prime}=\mathbb{k}\left[x_{1,1}^{ \pm 1}, \ldots, x_{1, k_{1}}^{ \pm 1}, x_{2,1}^{ \pm 1}, \ldots, x_{2, k_{2}}^{ \pm 1}, \ldots, x_{s, 1}^{ \pm 1}, \ldots, x_{s, k_{s}}^{ \pm 1}\right] .
$$

We consider the ideal $I^{\prime}=\operatorname{in}_{C}(I) S^{\prime}$ in $S^{\prime}$ and the variety $V\left(I^{\prime}\right)$ as a subvariety of $T^{k_{1}+\ldots+k_{s}}$. Then the proof proceeds as before.

Remark 2. From Lemma 1 we conclude the multiplicity being one is a necessary but not sufficient condition for toric initial ideals. A cone can have multiplicity one but its associated initial ideal might be neither prime nor binomial. There may be associated primes that contain monomials in the decomposition of $\operatorname{in}_{\mathbf{w}}(I)$ and these do not contribute to the multiplicity. We list examples of such cones in $\operatorname{trop}\left(\mathscr{F} \ell_{5}\right)$ as we will see in Theorem 5

Let $I$ be a homogeneous ideal in $S$ such that the Krull dimension of $S / I$ is $d$. Consider trop $(V(I)) \subset \mathbb{R}^{n+1} / H$ and the $d$-dimensional subfan $F \subset \mathbb{R}^{n+1}$ of the Gröbner fan of $I$ with $F / H \cong \operatorname{trop}(V(I))$. When $V(I) \subset \mathbb{P}^{k_{1}} \times \cdots \times \mathbb{P}^{k_{s}}$ the linear space $H$ is spanned by the rows of the matrix $D$. In particular, when $V(I) \subset \mathbb{P}^{n}$ we have that $H$ is equal to the span of $(1, \ldots, 1)$. We now describe some properties of the toric initial ideals corresponding to maximal cones of $\operatorname{trop}(V(I))$. Let $C$ be a cone in $\operatorname{trop}(V(I))$ and $\left\{\mathbf{w}_{1}, \ldots, \mathbf{w}_{d}\right\}$ be $d$ linearly independent vectors in $F$ generating the maximal cone $C^{\prime}$, such that $C^{\prime} / H \cong C$. We can assume that the $\mathbf{w}_{i}$ 's have integer entries since $F$ is a rational fan. We define the matrix associated to $C$ to be

$$
W_{C}=\left[\mathbf{w}_{1}, \ldots, \mathbf{w}_{d}\right]^{T}
$$

Consider a sublattice $L$ of $\mathbb{Z}^{n+1}$ and the standard basis $e_{1}, \ldots, e_{n+1}$ of $\mathbb{Z}^{n+1}$. Given $\ell=\left(\ell_{1}, \ldots, \ell_{n+1}\right) \in L$ we set $\ell^{+}=\sum_{\ell_{i}>0} \ell_{i} e_{i}$ and $\ell^{-}=-\sum_{\ell_{j}<0} \ell_{j} e_{j}$. Note that $\ell=$ $\ell^{+}-\ell^{-}$and $\ell^{+}, \ell^{-} \in \mathbb{N}^{n+1}$. We use here the same notation of [9] page 15].

Lemma 2. Let I be a homogeneous ideal in $S$ and $C$ a maximal cone in trop $(V(I))$. If $\operatorname{in}_{C}(I)$ is toric, then there exists a sublattice $L$ of $\mathbb{Z}^{n+1}$ and constants $0 \neq c_{\ell} \in \mathbb{k}$ with $\ell \in L$ such that

$$
\operatorname{in}_{C}(I)=I\left(W_{C}\right):=\left\langle x^{\ell^{+}}-c_{\ell} x^{\ell^{-}}: \ell \in L\right\rangle .
$$

In particular, $L$ is the kernel of the map $f: \mathbb{Z}^{n+1} \rightarrow \mathbb{Z}^{d}$ defined by the matrix $W_{C}$. If $C$ has multiplicity one and $\operatorname{in}_{C}(I)$ is not toric, then the unique toric ideal in the primary decomposition of $\operatorname{in}_{C}(I)$ is of the form $I\left(W_{C}\right)$.

Proof. Let $\operatorname{in}_{C}(I) \subset S$ be a toric initial ideal and let $C^{\prime}$ be the corresponding cone in $F$. The fan structure is defined on $\operatorname{trop}(V(I))$ so that for every $\mathbf{w}^{\prime}, \mathbf{w}$ in the relative interior of $C^{\prime}$ we have $\operatorname{in}_{\mathbf{w}^{\prime}}(I)=\operatorname{in}_{C}(I)=\operatorname{in}_{\mathbf{w}}(I)$. This implies $\operatorname{in}_{C}(I)$ is $W_{C^{-}}$ homogeneous with respect to the $\mathbb{Z}^{d}$-grading on $S$ given by the matrix $W_{C}$. By [31, Lemma 10.12] there exists an automorphism $\phi$ of $S$ sending $x_{i}$ to $\lambda_{i} x_{i}$ for some $\lambda_{i} \in \mathbb{k}$, such that the ideal $\operatorname{in}_{C}(I)$ is isomorphic to an ideal of the form

$$
I_{L}:=\left\langle\mathbf{x}^{\ell^{+}}-\mathbf{x}^{\ell^{-}}: \ell \in L\right\rangle .
$$


Here $L$ is the sublattice of $\mathbb{Z}^{n+1}$ given by the kernel of the map $f: \mathbb{Z}^{n+1} \rightarrow \mathbb{Z}^{d}$. Applying $\phi^{-1}$ to $\operatorname{in}_{C}(I)$ we can write each toric initial ideal as

$$
\left\langle\mathbf{x}^{\ell^{+}}-c_{\ell} \mathbf{x}^{\ell^{-}}: \ell \in L\right\rangle=I\left(W_{C}\right),
$$

for some $0 \neq c_{\ell} \in \mathbb{k}, L$ and $W_{C}$ defined above.

Let $C$ be a cone of multiplicity one and suppose $\operatorname{in}_{C}(I)$ is not prime. Then by Lemma 1 there exists a unique toric ideal $J$ in the primary decomposition of $\operatorname{in}_{C}(I)$. This toric ideal $J$ contains in ${ }_{C}(I)$ and we will show that it can be expressed as $I\left(W_{C}\right)$. The variety $V(I)$ is considered as subvariety of $\mathbb{P}^{n}$. As in Lemma1, the case $V(I) \subset$ $\mathbb{P}^{k_{1}} \times \cdots \times \mathbb{P}^{k_{s}}$ has an analogous proof.

The tropical variety depends only on the intersection of $V(I)$ with the torus, and $\operatorname{in}_{C}(I) \mathbb{k}\left[x_{1}^{ \pm 1}, \ldots, x_{n}^{ \pm 1}\right]$ is equal to $J$. Hence, $J$ is a prime ideal that is homogeneous with respect to $W_{C}$ so we can proceed as above to show $J$ can be written as $\left\langle\mathbf{x}^{\ell^{+}}-\right.$ $\left.c_{\ell} \mathbf{x}^{\ell^{-}}: \ell \in L\right\rangle=I\left(W_{C}\right)$.

Remark 3. Note that the lattice $L$ and the ideal $I\left(W_{C}\right)$ only depend on the linear space spanned by the rays of the cone $C^{\prime}$. Hence they are the same for every set of $d$ independent vectors in $C^{\prime}$ chosen to define $W_{C}$.

\section{Tropicalization and toric degenerations}

In this section we study the tropicalization of $\mathscr{F} \ell_{4}$ and $\mathscr{F} \ell_{5}$. We analyze the Gröbner toric degenerations arising from $\operatorname{trop}\left(\mathscr{F} \ell_{4}\right)$ and $\operatorname{trop}\left(\mathscr{F} \ell_{5}\right)$, and we compute the polytopes associated to their normalizations. In Proposition 1 we describe the tropical configurations arising from the maximal cones of trop $\left(\mathscr{F} \ell_{4}\right)$. These are configurations of a point on a tropical line in a tropical plane corresponding to the points in the relative interior of a maximal cone.

Before stating our main results, we recall the following definition.

Definition 6. There exists a unimodular equivalence between two lattice polytopes $P$ and $Q$ (resp. two fans $\mathscr{F}$ and $\mathscr{G}$ ) if there exists an affine lattice isomorphism $\phi$ of the ambient lattices sending the vertices (resp. the rays) of one polytope (resp. fan) to the vertices (resp. rays) of the other. Moreover, if $\sigma$ is a face of $P$ (resp. of $\mathscr{F}$ ) then $\phi(\sigma)$ is a face of $Q$ (resp. $\mathscr{G}$ ) and the adjacency of faces is respected.

Remark 4. We are interested in finding distinct fans up to unimodular equivalence as they give rise to non-isomorphic toric varieties. Often it will be possible only to determine combinatorial equivalence (see [9, §2.2]). This is a weaker condition but when it does not hold it allows us to rule out unimodular equivalence.

Theorem 4. The tropical variety $\operatorname{trop}\left(\mathscr{F} \ell_{4}\right)$ is a 6-dimensional rational fan in $\mathbb{R}^{14} / \mathbb{R}^{3}$ with a 3-dimensional lineality space. It consists of 78 maximal cones, 72 of which are prime. They are organized in five $S_{4} \rtimes \mathbb{Z}_{2}$-orbits, four of which contain prime cones. The prime cones give rise to four non-isomorphic toric degenerations. 
Proof. The theorem is proved by explicit computations. We developed a Macaulay 2 package called ToricDegenerations containing all the functions we use. The package and the data needed for this proof are available at

https ://github.com/ToricDegenerations.

The flag variety $\mathscr{F} \ell_{4}$ is a 6-dimensional subvariety of $\mathbb{P}^{3} \times \mathbb{P}^{5} \times \mathbb{P}^{3}$. The ideal $I_{4}$ defined in the previous section is contained in the total coordinate ring $R$ of $\mathbb{P}^{3} \times$ $\mathbb{P}^{5} \times \mathbb{P}^{3}$ which is the polynomial ring over $\mathbb{C}$ on the variables

$p_{1}, p_{2}, p_{3}, p_{4}, p_{12}, p_{13}, p_{14}, p_{23}, p_{24}, p_{34}, p_{123}, p_{124}, p_{134}, p_{234}$

The grading on $R$ is given by the matrix

$$
D=\left[\begin{array}{llllllllllllll}
1 & 1 & 1 & 1 & 0 & 0 & 0 & 0 & 0 & 0 & 0 & 0 & 0 & 0 \\
0 & 0 & 0 & 0 & 1 & 1 & 1 & 1 & 1 & 1 & 0 & 0 & 0 & 0 \\
0 & 0 & 0 & 0 & 0 & 0 & 0 & 0 & 0 & 0 & 1 & 1 & 1 & 1
\end{array}\right] .
$$

The explicit form of $I_{4}$ can be found in [26, page 276]. As we have seen in $₫ 2$ the tropicalization of $\mathscr{F} \ell_{4}$ is contained in $\mathbb{R}^{14} / H$. In this case $H$ is the vector space spanned by the rows of $D$.

We use the Macaulay2 [19] interface to Gfan [21] to compute trop $\left(\mathscr{F} \ell_{4}\right)$. The given input is the ideal $I_{4}$ and the $S_{4} \rtimes \mathbb{Z}_{2}$-action (see [22, §3.1.1]). The output is a subfan $F$ of the Gröbner fan of dimension 9 . We quotient it by $H$ to get $\operatorname{trop}\left(\mathscr{F} \ell_{4}\right)$ as a 6-dimensional fan contained in $\mathbb{R}^{14} / H \cong \mathbb{R}^{14} / \mathbb{R}^{3}$.

Firstly, the function computeWeightVectors computes a list of vectors. There is one for every maximal cone of trop $\left(\mathscr{F} \ell_{4}\right)$ and it is contained in the relative interior of the corresponding cone. Then groebnerToricDegenerations computes all the initial ideals and checks if they are binomial and prime over $\mathbb{Q}$. These are organized in a hash table, which is the output of the function. All 78 initial ideals are binomial and all maximal cones have multiplicity one. In order to check primeness over $\mathbb{C}$, we have to check if $\operatorname{in}_{C}\left(I_{4}\right)=I\left(W_{C}\right)$. This can be done by computing the degrees of $V\left(\operatorname{in}_{C}\left(I_{4}\right)\right)$ and $V\left(I\left(W_{C}\right)\right)$ seen as subvarieties of $\mathbb{P}^{13}$. If these are equal, then there are no non-toric ideals in the primary decomposition of $\operatorname{in}_{C}\left(I_{4}\right)$. Note that the degree of $V\left(I\left(W_{C}\right)\right)$ equals the degree of $V\left(I_{L}\right)$, where $L$ and $I_{L}$ can be computed from $W_{C}$ as in the proof of Lemma2,

We consider the orbits of the $S_{4} \ltimes \mathbb{Z}_{2}$-action on the set of initial ideals. These correspond to the orbits of maximal cones of $F$ and hence of $\operatorname{trop}\left(\mathscr{F} \ell_{4}\right)$. There is one orbit of non-prime initial ideals and four orbits of prime initial ideals. The varieties corresponding to initial ideals contained in the same orbit are isomorphic. Therefore, for each orbit we choose a representative of the form in ${ }_{C}\left(I_{4}\right)=I\left(W_{C}\right)$ for some cone $C$.

We now compute for each of the four prime orbits, the polytope of the normalization of the associated toric varieties. We use the Macaulay2-package Polyhedra [4] for the following computations. 
The lattice $M$ associated to $S / I\left(W_{C}\right)$ is generated over $\mathbb{Z}$ by the columns of $W_{C}$. To use Polyhedra we want to have a lattice with index 1 in $\mathbb{Z}^{9}$. Hence, in case the index of $M$ in $\mathbb{Z}^{9}$ is different from 1 , we consider $M$ as the lattice generated by the columns of the matrix $\left(\operatorname{ker}\left(\left(\operatorname{ker}\left(W_{C}\right)\right)^{T}\right)^{T}\right.$. Here, for a matrix $A$ we consider $\operatorname{ker}(A)$ to be the matrix whose columns minimally generate the kernel of the map $\mathbb{Z}^{14} \rightarrow \mathbb{Z}^{9}$ defined by $A$. We denote the set of generators of $M$ by $\mathscr{B}_{C}=\left\{\mathbf{b}_{1}, \ldots, \mathbf{b}_{14}\right\}$ so that $M=\mathbb{Z} \mathscr{B}_{C}$.

The toric variety $\mathbb{P}^{3} \times \mathbb{P}^{5} \times \mathbb{P}^{3}$ can be seen as $\operatorname{Proj}\left(\oplus_{\ell} R_{\ell(1,1,1)}\right)$ and $I\left(W_{C}\right)$ as an ideal in $\oplus_{\ell} R_{\ell(1,1,1)}$ (see [26, Chapter 10]). The associated toric variety is $\operatorname{Proj}\left(\oplus_{\ell} \mathbb{C}\left[\mathbb{N} \mathscr{B}_{C}\right]_{\ell(1,1,1)}\right)$. The polytope $P$ of the normalization is given as the convex hull of those lattice points in $\mathbb{N}_{C}$ corresponding to degree $(1,1,1)$-monomials in $\mathbb{C}\left[\mathbb{N} \mathscr{B}_{C}\right]$.

These can be found in the following way. We order the rows of the matrix $\left(\mathbf{b}_{1}, \ldots, \mathbf{b}_{14}\right)$ associated to $\mathscr{B}_{C}$ so that the first three rows give the matrix $D$ from (2). Now the matrix $\left(\mathbf{b}_{1}, \ldots, \mathbf{b}_{14}\right)$ represents a map $\mathbb{Z}^{14} \rightarrow \mathbb{Z}^{3} \oplus \mathbb{Z}^{6}$, where $\mathbb{Z}^{3} \oplus \mathbb{Z}^{6}$ is the lattice $M$ and the $\mathbb{Z}^{3}$ part gives the degree of the monomials associated to each lattice point on $M$. The lattice points, whose convex hull give the polytope $P$, are those ones with the first three coordinates being 1 . In other words, we have obtained $P$ by applying the reverse procedure of constructing a toric variety from a polytope (see [9, §2.1-§2.2]). Note that the difference from the procedure given in [9, §2.1$\S 2.2]$ is the $\mathbb{Z}^{3}$-grading and because of that we do not consider the convex hull of $\mathscr{B}_{C}$, but the intersection of $\mathbb{N} \mathscr{B}_{C}$ with these hyperplanes.

In Table 1 there are the numerical invariants of the initial ideals and their corresponding polytopes. Using polymake [17] we first obtain that there is no combinatorial equivalence between each pair of polytopes. This means that there is no unimodular equivalence between the corresponding normal fans, hence the normalization of the toric varieties associated to these toric degenerations are not isomorphic. This implies that we obtain four non-isomorphic toric degenerations.

\begin{tabular}{|l|l|l|l|l|l|}
\hline Orbit & Size & Cohen-Macaulay & Prime & $\#$ Generators & F-vector of associated polytope \\
\hline 1 & 24 & Yes & Yes & 10 & $(42,141,202,153,63,13)$ \\
2 & 12 & Yes & Yes & 10 & $(40,132,186,139,57,12)$ \\
3 & 12 & Yes & Yes & 10 & $(42,141,202,153,63,13)$ \\
4 & 24 & Yes & Yes & 10 & $(43,146,212,163,68,14)$ \\
5 & 6 & Yes & No & 10 & Not applicable \\
\hline
\end{tabular}

Table 1 The tropical variety trop $\left(\mathscr{F} \ell_{4}\right)$ has 78 maximal cones organized in five $S_{4} \rtimes \mathbb{Z}_{2}$-orbits. The algebraic invariants of the initial ideals associated to these cones and the F-vectors of their associated polytopes are listed here.

Proposition 1. There are six tropical configurations up to symmetry (depicted in Figure 21) arising from the maximal cones of trop $\left(\mathscr{F} \ell_{4}\right)$. They are further organized in five $S_{4} \rtimes \mathbb{Z}_{2}$-orbits. 
Proof. The tropical variety trop $\left(\mathscr{F} \ell_{4}\right)$ is contained in

$$
\operatorname{trop}\left(\operatorname{Gr}\left(1, \mathbb{C}^{4}\right)\right) \times \operatorname{trop}\left(\operatorname{Gr}\left(2, \mathbb{C}^{4}\right)\right) \times \operatorname{trop}\left(\operatorname{Gr}\left(3, \mathbb{C}^{4}\right)\right) .
$$

Each tropical Grassmannian parametrizes tropicalized linear spaces (see [25, Theorem 4.3.17]). This implies that every point $p$ in trop $\left(\mathscr{F} \ell_{4}\right)$ corresponds to a chain of tropical linear subspaces given by a point on a tropical line contained in a tropical plane. All tropical chains are realizable, meaning that they are the tropicalization of the classical chains of linear spaces of $\mathbb{k}^{4}$ corresponding to a point $q$ in $\mathscr{F} \ell_{4}$ such that $\mathfrak{v}(q)=p$, where $\mathbb{k}=\mathbb{C}\{\{t\}\}$ and $\mathfrak{v}$ is the natural valuation on this field (see [25, Part (3) of Theorem 3.2.3]).

In this case, there is only one combinatorial type for the tropical plane and four possible types for the lines up to symmetry (see [25, Example 4.4.9]). The plane consists of six 2-dimensional cones positively spanned by all possible pairs of vectors $(1,0,0)^{T},(0,1,0)^{T},(0,0,1)^{T}$, and $(-1,-1,-1)^{T}$. The combinatorial types of the tropical lines are shown in Figure 1. The leaves of these graphs represent the rays of the tropical line labeled 1 up to 4 corresponding to the positive hull of each of the vectors $(1,0,0)^{T},(0,1,0)^{T},(0,0,1)^{T}$, and $(-1,-1,-1)^{T}$.

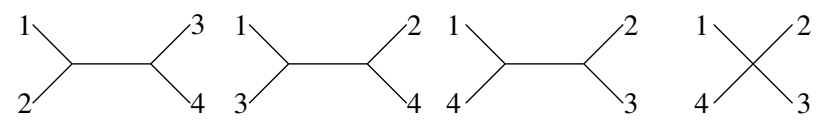

Fig. 1 Combinatorial types of tropical lines in $\mathbb{R}^{4} / \mathbb{R} \mathbf{1}$.

Consider the $S_{4} \rtimes \mathbb{Z}_{2}$-orbits of maximal cones of trop $\left(\mathscr{F} \ell_{4}\right)$. If we compute the chain of tropical linear spaces corresponding to an element in each orbit, we get the configurations in Figure 2 Note that we do not include the labeling since up to symmetry we can get all possibilities. The point on the line is the black dot. In case the intersection of the line with the rays of the plane is the vertex of the plane then we denote this with a hollow dot. A vertex of the line is colored in gray if it lies on a ray of the plane. For example in orbit 2, label the rays 1 to 4 anti-clockwise starting from the top left edge. We have rays 1 and 2 in the 2-dimensional positive hull of $(1,0,0)^{T}$ and $(0,1,0)^{T}$. The vector associated to the internal edge is $(1,1,0)^{T}$. The gray point is the origin and the black point has coordinates $(a, 1,0)^{T}$ for $a>1$.

Orbits 1 and 4 in Figure 2 have size 24, orbits 2 and 3 have size 12 and orbit 5 has size 6 . Note that orbit 5 corresponds to non-prime initial ideals. Orbit 1 contains two combinatorial types of tropical configurations and one is sent to the other by the $\mathbb{Z}_{2}$-action on the tropical variety. The orbits 2 and 3 differ from the fact that for each combinatorial type of line the gray dot can lie on one of the four rays of the tropical plane. These possibilities are grouped in two pairs, one is in orbit 2 and the other in orbit 3. 
Orbit 1
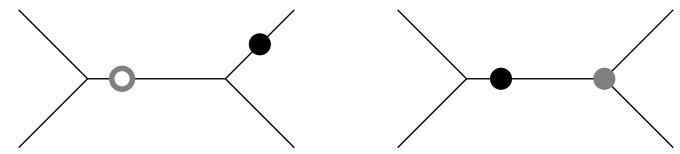

Orbit 2

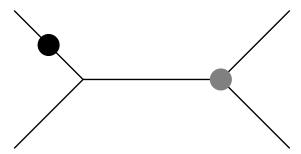

Orbit 3

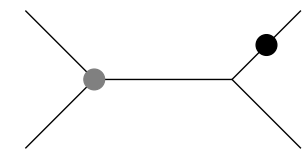

Orbit 4
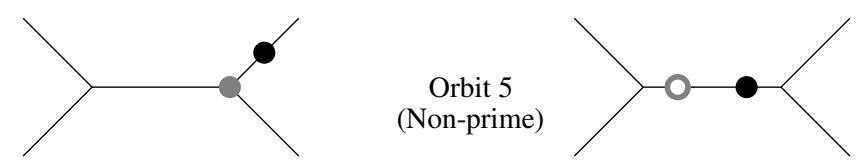

the vertex of the plane

a point on a ray of the plane

- the point

Fig. 2 The list of all tropical configurations up to symmetry that arise in $\mathscr{F} \ell_{4}$. The hollow and the full gray dot denote whether that vertex of the line is the vertex of the plane or it is contained in a ray of the plane. The black dot is the position of the point on the line.

Theorem 5. The tropical variety trop $\left(\mathscr{F} \ell_{5}\right)$ is a 10 -dimensional fan in $\mathbb{R}^{30} / \mathbb{R}^{4}$ with a 4-dimensional lineality space. It consists of 69780 maximal cones which are grouped in $536 S_{5} \rtimes \mathbb{Z}_{2}$-orbits. These give rise to 531 orbits of binomial initial ideals and among these 180 are prime. They correspond to 180 non-isomorphic toric degenerations.

Proof. The flag variety $\mathscr{F} \ell_{5}$ is a 10 -dimensional variety defined by 66 quadratic polynomials in the total coordinate ring of $\mathbb{P}^{4} \times \mathbb{P}^{9} \times \mathbb{P}^{9} \times \mathbb{P}^{4}$. These are of the form $\sum_{j \in J \backslash I}(-1)^{l_{j}} p_{I \cup\{j\}} p_{J \backslash\{j\}}$, where $J, I \subset\{1, \ldots, 5\}$ and $l_{j}=\#\{k \in J: j<k\}+\#\{i \in$ $I: i<j\}$.

The proof is similar to the proof of Theorem 4 The only difference is that the action of $S_{5} \rtimes \mathbb{Z}_{2}$ on $\mathscr{F} l_{5}$ is crucial for the computations. In fact, without exploiting the symmetries the calculations to get the tropicalization would not terminate. Moreover, we only verify primeness of the initial ideals over $\mathbb{Q}$ using the primdec library [28] in Singular [10]. We compute the polytopes associated to the normalization of the 180 toric varieties in the same way as Theorem 4, only changing the matrix of the grading. It is now given by

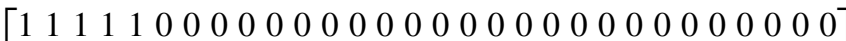

$$
\begin{aligned}
& 0000011111111110000000000000000 \\
& 0000000000000000001111111111000000
\end{aligned}
$$

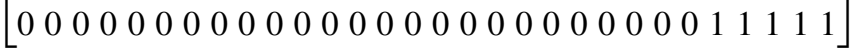


Since there are no combinatorial equivalences among the normal fans to these polytopes, we deduce that the obtained toric degenerations are pairwise non-isomorphic. More information on the non-prime initial ideals is available in Table 4 in the appendix.

\section{String polytopes and the FFLV-polytope}

This section provides an introduction to string cones, string polytopes, and the FFLV polytope with explicit computations for $\mathscr{F} \ell_{4}$ and $\mathscr{F} \ell_{5}$. String polytopes are described by Littelmann in [24], and by Berenstein and Zelevinsky in [3]. FFLV stands for Feigin, Fourier, and Littelmann, who defined this polytope in [15], and Vinberg who conjectured its existence in a special case. Both, the string polytopes and the FFLV polytope, can be used to obtain toric degenerations of the flag variety.

Let $W=S_{n}$ be the symmetric group, which is the Weyl group corresponding to $G=S L_{n}$ over $\mathbb{C}$ with the longest word $w_{0}$ given in the alphabet of simple transpositions $s_{i}=(i, i+1) \in S_{n}$. We choose the Borel subgroup $B \subset S L_{n}$ of upper triangular matrices and the maximal torus $T \subset B$ of diagonal matrices. Further, let $U^{-} \subset B^{-}$be the unipotent radical in the opposite Borel subgroup, i.e. the set of lower triangular matrices with 1's on the diagonal. Let $\operatorname{Lie}(G)=\mathfrak{g}=\mathfrak{s l}_{n}$ be the corresponding Lie algebra, i.e. $n \times n$-matrices with trace zero. Let $\mathfrak{h}=\operatorname{Lie}(T) \subset \mathfrak{g}$ be diagonal matrices. We fix a Cartan decomposition $\mathfrak{g}=\mathfrak{n}^{-} \oplus \mathfrak{b}$ with $\operatorname{Lie}(B)=\mathfrak{b}$ and $\operatorname{Lie}\left(U^{-}\right)=\mathfrak{n}^{-}$. Note that $S L_{n} / B=\mathscr{F} \ell_{n}$. By $R$ we denote the root system of $\mathfrak{g}$ (see [20, Section 9.2] for the definition). Here $R$ is of type $\mathrm{A}_{n-1}$. Let $R^{+}$be the set of positive roots with respect to the given choice of $\mathfrak{b}$. We denote the simple roots generating the root lattice by $\alpha_{1}, \ldots, \alpha_{n-1}$, and their coroots generating the dual lattice by $\alpha_{i}^{\vee}$. For positive roots $\alpha_{i}+\alpha_{i+1}+\cdots+\alpha_{j}$ with $j \geq i$ we use the short notation $\alpha_{i, j}$. Note that using this notation we have $\alpha_{i, i}=\alpha_{i}$. The number of positive roots is $N$, which is also the length of $w_{0}$ as reduced expression in the $s_{i}$. For a positive $\operatorname{root} \beta \in R^{+}$, $f_{\beta}$ is a non-zero root vector in $\mathfrak{n}^{-}$of weight $-\beta$. Let $P$ denote the weight lattice of $T$ generated by the fundamental weights $\omega_{1}, \ldots, \omega_{n-1}$. The definition can be found in [20, Section 13.1]. A weight $\lambda \in P$ is regular dominant, if $\lambda=\sum_{i=1}^{n-1} a_{i} \omega_{i}$ with $a_{i} \in \mathbb{Z}_{>0}$ for all $i$. The subset of regular dominant weights is denoted by $P^{++}$.

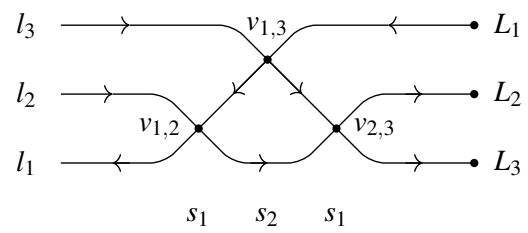

Fig. 3 Pseudoline arrangement corresponding to $\underline{w}_{0}=s_{1} s_{2} s_{1}$ for $\mathscr{F} \ell_{3}$ with orientation induced by $l_{1}$. 
For a fixed weight $\lambda \in P^{++}$and a reduced expression $\underline{w}_{0}$ of $w_{0}$ we construct the string polytope $Q_{w_{0}}(\lambda)$. This description can be found in [18] and [24]. To $\underline{w}_{0}$ one associates a pseudoline arrangement. It consists of $n$ horizontal pseudolines (or in short lines) labeled 1 to $n$ on the left from bottom to top. Pairwise, they cross exactly once and the order of crossings depends on $\underline{w}_{0}$. More precisely, a simple reflection $s_{i}$ induces a crossing on level $i$, see Figure 3 . The diagram has vertices $v_{i, j}$ for every crossing of lines $l_{i}$ and $l_{j}$, as well as vertices $L_{1}, \ldots, L_{n}$ from top to bottom at the right ends of the lines. Every line $l_{i}$ with $1 \leq i<n$ induces an orientation of the diagram obtained by orienting $l_{j}$ for $j>i$ from left to right and $l_{k}$ for $k \leq i$ from right to left.

Fix an oriented path $v_{0} \rightarrow \cdots \rightarrow v_{s}$ in an (oriented) pseudoline arrangement and assume three adjacent vertices $v_{k-1} \rightarrow v_{k} \rightarrow v_{k+1}$ on the path belong to the same pseudoline $l_{i}$. Whenever a path does not change the line at a crossing, we are in this situation. Let $v_{k}$ be the intersection of $l_{i}$ and $l_{j}$. The path is rigorous, if it avoids the following two situations:

- $i<j$ and both lines are oriented to the left or

- $i>j$ and both lines are oriented to the right.

The first situation is visualized on the left of Figure 4 and the second on the right. The thick arrow is the part of line $l_{i}$ that must not be contained in a rigorous path. We denote by $\mathscr{P}_{\underline{w}_{0}}$ the set of all possible rigorous paths for all orientations induced by the lines $l_{i}$ with $1 \leq i<n$.
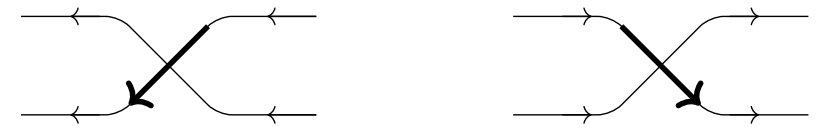

Fig. 4 The two local orientations with thick arrows forbidden in rigorous paths.

Example 1. Consider $\mathscr{F} \ell_{4}$ with reduced expression $\underline{w}_{0}=s_{1} s_{2} s_{3} s_{2} s_{1} s_{2}$. We draw the corresponding pseudoline arrangement in Figure 5 with orientation induced by $l_{1}$. The rigorous paths for this orientation have source $L_{1}$ and sink $L_{2}$. An example of a rigorous path is

$$
\mathbf{p}=L_{1} \rightarrow v_{1,4} \rightarrow v_{1,3} \rightarrow v_{3,4} \rightarrow v_{2,3} \rightarrow L_{2}
$$

An example for a non-rigorous path is one that passes through a thick arrow, for example

$$
\mathbf{p}^{\prime}=L_{1} \rightarrow v_{1,4} \rightarrow v_{3,4} \rightarrow v_{2,4} \rightarrow v_{2,3} \rightarrow L_{2} .
$$

Back to the general case, we fix an orientation induced by $l_{i}, 1 \leq i<n$ and consider all rigorous paths from $L_{i}$ to $L_{i+1}$. We associate the weight $c_{\mathbf{p}}$ to each such path $\mathbf{p}$ as follows. Denote by $\left\{c_{i, j}\right\}_{1 \leq i, j \leq n}$ the standard basis of $\mathbb{R}^{N}$, where we set $c_{i, j}=-c_{j, i}$ if $i>j$ and $c_{j, j}=0$. Note that $N$ is the number of crossings in a pseudoline arrangement and hence we can associate the basis vector $c_{i, j}$ to the crossing of 


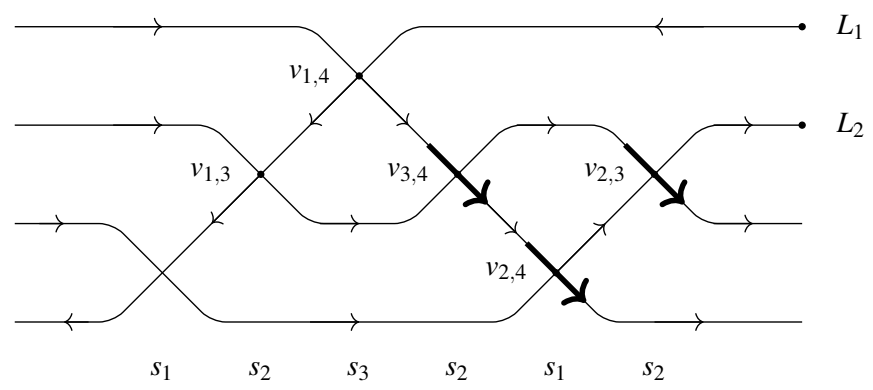

Fig. 5 A pseudoline arrangement for $\mathscr{F} \ell_{4}$ with $\underline{w}_{0}=s_{1} s_{2} s_{3} s_{2} s_{1} s_{2}$ and orientation induced by $l_{1}$. Thick arrows denote forbidden line segments for rigorous paths.

$l_{i}$ and $l_{j}$ for $1 \leq i, j \leq n$. Consider a rigorous path $\mathbf{p}=L_{i} \rightarrow v_{r_{1}} \rightarrow \cdots \rightarrow v_{r_{m}} \rightarrow L_{i+1}$. Every vertex $v_{r_{s}}$ corresponds to the crossing of two lines $l_{k}$ and $l_{j}$. If $\mathbf{p}$ changes from line $l_{k}$ to line $l_{j}$ at $v_{r_{s}}$ we associate the vector $c_{k, j} \in \mathbb{R}^{N}$. We set $c_{\mathbf{p}}$ to be the sum of all such $c_{k, j}$ in $\mathbf{p}$ and denote it by $c_{\mathbf{p}}$.

Definition 7. For a fixed reduced expression $\underline{w}_{0}$, we define the string cone to be

$$
C_{\underline{w}_{0}}=\left\{\left(y_{i, j}\right) \in \mathbb{R}^{N} \mid\left(c_{\mathbf{p}}\right)^{T}\left(y_{i, j}\right) \geq 0, \forall \mathbf{p} \in \mathscr{P}_{\underline{w}_{0}}\right\} .
$$

This is not the original definition of a string cone, but an equivalent one (see [18, Corollary 5.8]). It can be extended to describe string cones for Schubert varieties, see [6].

Example 2. There are two rigorous paths in Figure 3, $L_{1} \rightarrow v_{1,3} \rightarrow v_{2,3} \rightarrow L_{2}$ and $L_{1} \rightarrow v_{1,3} \rightarrow v_{1,2} \rightarrow v_{2,3} \rightarrow L_{2}$. The corresponding weights are $c_{1,3}-c_{2,3}$ and $c_{1,2}$ inducing the inequalities $y_{1,3}-y_{2,3} \geq 0$ and $y_{1,2} \geq 0$. Considering the orientation induced by $l_{2}$ there is a rigorous path $L_{2} \rightarrow v_{2,3} \rightarrow L_{3}$ which gives the inequality $y_{2,3} \geq 0$. The string cone corresponding to the underlying non-oriented pseudoline arrangement in Figure 3 is then given by

$$
C_{S_{1} s_{2} s_{1}}=\left\{y_{1,2} \geq 0, y_{1,3} \geq y_{2,3} \geq 0\right\} .
$$

Each crossing of lines $l_{k}$ and $l_{m}$ corresponds to an index $i_{j}$ associated to a simple reflection $s_{i_{j}}$ in $\underline{w}_{0}$ (see e.g. Figure 3). We will therefore also denote $c_{k, m}=c_{j}$. Let $1 \leq i \leq n-1$ and $r_{1}, \ldots, r_{n_{i}}$ be the indices such that $s_{i_{r_{p}}}=s_{i}$ in $\underline{w}_{0}$ for $1 \leq$ $p \leq n_{i}$. Further, let $k_{1}, \ldots, k_{t}$ be the positions where $s_{i_{k_{m}}} \in\left\{s_{i-1}, s_{i+1}\right\}$ for $1 \leq m \leq$ $t$. In particular, $r_{1}, \ldots, r_{n_{i}}$ are those positions inducing a crossing at level $i$ in the corresponding pseudoline arrangement. The following appears in [24].

Definition 8. The weighted string cone $\mathscr{C}_{\underline{w}_{0}} \subset \mathbb{R}^{N} \times \mathbb{R}_{>0}^{n-1}$ is obtained from $C_{\underline{w}_{0}}$ by adding variables $m_{1}, \ldots, m_{n-1}$, and for every $1 \leq i \leq n-1$ and $j \in\left\{r_{1}, \ldots, r_{n_{i}}\right\}$ the inequality 


$$
m_{i}-y_{j}-2 \sum_{r_{p}>j} y_{r_{p}}+\sum_{k_{p}>j} y_{k_{p}} \geq 0
$$

where $\left(y_{k}, m_{l}\right)_{\substack{1 \leq k \leq N \\ 1 \leq l<n}} \in \mathbb{R}^{N} \times \mathbb{R}_{\geq 0}^{n-1}$. For a weight $\lambda=\sum_{i=1}^{n-1} a_{i} \omega_{i} \in P$ the string polytope is defined as

$$
Q_{\underline{w}_{0}}(\lambda):=Q_{\underline{w}_{0}} \cap H_{\lambda} .
$$

Here $H_{\lambda}$ is the intersection of the hyperplanes defined by $m_{i}=a_{i}$ for all $1 \leq i<n$.

The additional weight inequalities can also be obtained combinatorially as described in [6]. We will consider for all computations the weight $\rho=\sum_{i=1}^{n-1} \omega_{i}$. This is the weight in $P^{++}$with minimal choice of coefficients of fundamental weights in $\mathbb{Z}_{>0}$, namely all are 1 . Note that all string polytopes are cut out from the weighted string cone, but for different weights they are different polytopes.

The following result is a simplified version of Theorem 1 proven by Caldero [7] for flag varieties. A more general statement is given by Alexeev and Brion in [1, Theorem 3.2].

Theorem 6. There exists a flat family $\mathscr{X} \rightarrow \mathbb{A}^{1}$ for a normal variety $\mathscr{X}$ such that for $t \neq 0$ the fiber over $t$ is isomorphic to $\mathscr{F} \ell_{n}$ and for $t=0$ it is isomorphic to a projective toric variety $X_{0}$ with polytope $Q_{\underline{w}_{0}}(\lambda)$ for $\lambda \in P^{++}$.

The proof of Theorem 6 uses the embedding $\mathscr{F} \ell_{n} \hookrightarrow \mathbb{P}(V(\lambda))$ and Lusztig's dual canonical basis, where $V(\lambda)$ is the irreducible representation of $\mathfrak{s l}_{n}$ with highest weight $\lambda$.

For two polytopes $A, B \subset \mathbb{R}^{l}$, the Minkowski sum is defined to be $A+B=\{a+b$ : $a \in A, b \in B\}$. Consider the weight $\rho$. The string polytope $Q_{\underline{w}_{0}}(\rho)$ is in general not the Minkowski sum of string polytopes $Q_{\underline{w}_{0}}\left(\omega_{1}\right), \ldots, Q_{\underline{w}_{0}}\left(\omega_{n-1}\right)$, which motivates the following definition.

Definition 9. A string cone has the weak Minkowski property (MP), if for every lattice point $p \in Q_{\underline{w}_{0}}(\rho)$ there exist lattice points $p_{\omega_{i}} \in Q_{\underline{w}_{0}}\left(\omega_{i}\right)$ such that

$$
p=p_{\omega_{1}}+p_{\omega_{2}}+\cdots+p_{\omega_{n-1}} .
$$

Remark 5. Note that the (non-weak) Minkowski property would require the above condition on lattice points to be true for arbitrary weights $\lambda$. Further, note that if $Q_{\underline{w}_{0}}(\rho)$ is the Minkowski sum of the fundamental string polytopes $Q_{\underline{w}_{0}}\left(\omega_{i}\right)$, then MP is satisfied.

Proposition 2. For $\mathscr{F} \ell_{4}$ there are four string polytopes in $\mathbb{R}^{10}$ up to unimodular equivalence and three of them satisfy MP. For $\mathscr{F} \ell_{5}$ there are 28 string polytopes in $\mathbb{R}^{14}$ up to unimodular equivalence and 14 of them satisfy $M P$.

Proof. We first consider $\mathscr{F} \ell_{4}$. There are 16 reduced expressions for $w_{0}$. Simple transpositions $s_{i}$ and $s_{j}$ with $1 \leq i<i+1<j<n$ commute and are also called orthogonal. We consider reduced expressions up to changing those, i.e. there are 
eight symmetry classes. We fix the weight in $P^{++}$to be $\rho=\omega_{1}+\omega_{2}+\omega_{3}$. The string polytopes are organized in four classes up to unimodular equivalence. See Table 2] in which 121321 denotes the reduced expression $\underline{w}_{0}=s_{1} s_{2} s_{1} s_{3} s_{2} s_{1}$. Hence they give four different toric degenerations for the embedding $\mathscr{F} \ell_{4} \hookrightarrow \mathbb{P}(V(\rho))$.

\begin{tabular}{|c|c|c|c|c|c|}
\hline$\overline{w_{0}}$ & Normal & MP & Weight vector $\mathbf{w}_{w_{0}}$ & Prime & Tropical cone \\
\hline $\begin{array}{l}\text { String 1: } \\
121321 \\
212321 \\
232123 \\
323123\end{array}$ & $\begin{array}{l}\text { yes } \\
\text { yes } \\
\text { yes } \\
\text { yes }\end{array}$ & $\begin{array}{l}\text { yes } \\
\text { yes } \\
\text { yes } \\
\text { yes }\end{array}$ & $\begin{array}{l}(0,32,24,7,0,16,6,48,38,30,0,4,20,52) \\
(0,16,48,7,0,32,6,24,22,54,0,4,36,28) \\
(0,4,36,28,0,32,24,6,22,54,0,16,48,7) \\
(0,4,20,52,0,16,48,6,38,30,0,32,24,7)\end{array}$ & $\begin{array}{l}\text { yes } \\
\text { yes } \\
\text { yes } \\
\text { yes }\end{array}$ & $\begin{array}{l}\text { rays } 10,18,19, \text { cone } 71 \\
\text { rays } 6,10,19, \text { cone } 44 \\
\text { rays } 0,3,6, \text { cone } 3 \\
\text { rays } 0,1,3, \text { cone } 1\end{array}$ \\
\hline $\begin{array}{l}\text { String 2: } \\
123212 \\
321232\end{array}$ & $\begin{array}{l}\text { yes } \\
\text { yes }\end{array}$ & $\begin{array}{l}\text { yes } \\
\text { yes }\end{array}$ & $\begin{array}{l}(0,32,18,14,0,16,12,48,44,27,0,8,24,56) \\
(0,8,24,56,0,16,48,12,44,27,0,32,18,14)\end{array}$ & $\begin{array}{l}\text { yes } \\
\text { yes }\end{array}$ & $\begin{array}{l}\text { rays } 2,10,18 \text {, cone } 36 \\
\text { rays } 0,1,2 \text {, cone } 0\end{array}$ \\
\hline $\begin{array}{l}\text { String 3: } \\
213231\end{array}$ & yes & yes & $(0,16,48,13,0,32,12,20,28,60,0,8,40,22)$ & yes & rays $3,6,19$, cone 24 \\
\hline $\begin{array}{l}\text { String 4: } \\
132312\end{array}$ & yes & no & $(0,16,12,44,0,8,40,24,56,15,0,32,10,26)$ & no & rays $1,2,17$, cone 17 \\
\hline FFLV & yes & yes & $\begin{array}{l}w^{\text {min }}=(0,2,2,1,0,1,1,2,1,2,0,1,1,1) \\
w^{\text {reg }}=(0,3,4,3,0,2,2,4,3,5,0,1,2,3)\end{array}$ & yes & $\begin{array}{l}\text { rays } 9,11,12, \text { cone } 56 \\
\text { rays } 9,11,12, \text { cone } 56\end{array}$ \\
\hline
\end{tabular}

Table 2 Isomorphism classes of string polytopes for $n=4$ and $\rho$ depending on $\underline{w}_{0}$, normality, the weak Minkowsky property, the weight vectors $\mathbf{w}_{\underline{w}_{0}}$ constructed in 5 primeness of the binomial initial ideals $\operatorname{in}_{\mathbf{w}_{w_{0}}}\left(I_{4}\right)$, and the corresponding tropical cones with their spanning rays as they appear at http://www.mi.uni-koeln.de/ lbossing/tropflag/tropflag4.html.

In order to verify whether the weak Minkowski property holds or not, we proceed as follows. We fix $\underline{w}_{0}$ to compute the string polytope $Q_{\underline{w}_{0}}(\rho)$ using polymake. The number of lattice points in $Q_{\underline{w}_{0}}(\rho)$ is $\operatorname{dim}(V(\rho))=64$. Then we compute the polytopes $Q_{\underline{w}_{0}}\left(\omega_{1}\right), Q_{\underline{w}_{0}}\left(\omega_{2}\right), Q_{\underline{w}_{0}}\left(\omega_{3}\right)$ and set $P=Q_{\underline{w}_{0}}\left(\omega_{1}\right)+Q_{\underline{w}_{0}}\left(\omega_{2}\right)+Q_{\underline{w}_{0}}\left(\omega_{3}\right)$. Now let $L P(P)$ be the set of lattice points in $P$. If $|L P(P)|<64$, then there exists a lattice point in $Q_{\underline{w}_{0}}(\rho)$, that can not be expressed as $p_{1}+p_{2}+p_{3}$ for $p_{i} \in Q_{\underline{w}_{0}}\left(\omega_{i}\right)$. For $\underline{w}_{0}=s_{1} s_{3} s_{2} s_{3} s_{1} s_{2}$, we observe that

$$
\left|L P\left(Q_{\underline{w}_{0}}\left(\omega_{1}\right)+Q_{\underline{w}_{0}}\left(\omega_{2}\right)+Q_{\underline{w}_{0}}\left(\omega_{3}\right)\right)\right|=62<64 .
$$

Hence the class String 4 does not satisfy MP. For the classes String 1, 2, and 3 equality holds and MP is satisfied.

Now consider $\mathscr{F} l_{5}$. There are 62 reduced expressions $\underline{w}_{0}$ up to changing orthogonal transpositions. The map $L: S_{5} \rightarrow S_{5}$ given on simple reflections by $L\left(s_{i}\right)=s_{4-i+1}$ induces a symmetry among the string polytopes. Namely, for a fixed $\lambda \in P^{++}$, there is a unimodular equivalence between $Q_{\underline{w}_{0}}(\lambda)$ and $Q_{L\left(\underline{w}_{0}\right)}(\lambda)$. Exploiting this symmetry, we compute 31 string polytopes for $\rho$. These are organized in 28 unimodular equivalence classes, that arise from further symmetries of the underlying pseudoline arrangements. Table 6 shows which reduced expressions belong to string polytopes within one class of unimodular equivalence, and which string cones satisfy MP. Proceeding as for $\mathscr{F} \ell_{4}$, we observe that 14 out of 28 classes satisfy MP. 
We will now turn to the FFLV polytope. It is defined in [15] by Feigin, Fourier, and Littelmann to describe bases of irreducible highest weight representations $V(\lambda)$. In [16] they give a construction of a flat degeneration of the flag variety into the toric variety associated to the FFLV polytope. It is also an example of the more general setup presented in [12]. We give the general definition here and compute the FFLV polytopes for $\mathscr{F} \ell_{4}$ and $\mathscr{F} \ell_{5}$ for $\rho$. Recall, that $\alpha_{i}$ for $1 \leq i<n$ are the simple roots of $\mathfrak{s l}_{n}$, and $\alpha_{p, q}$ is the positive root $\alpha_{p}+\alpha_{p+1}+\cdots+\alpha_{q}$ for $1 \leq p \leq q<n$.

Definition 10. A Dyck path is a sequence of positive roots $\mathbf{d}=\left(\beta_{0}, \ldots, \beta_{k}\right)$ with $k \geq 0$ satisfying the following conditions

1. if $k=0$ then $\mathbf{d}=\left(\alpha_{i}\right)$ for $1 \leq i \leq n-1$,

2. if $k \geq 1$ then

a. the first and the last roots are simple, i.e. $\beta_{0}=\alpha_{i}, \beta_{k}=\alpha_{j}$ for $1 \leq i<j \leq n-1$,

b. if $\beta_{s}=\alpha_{p, q}$ then $\beta_{s+1}$ is either $\alpha_{p, q+1}$ or $\alpha_{p+1, q}$.

Denote by $\mathscr{D}$ the set of all Dyck paths. We choose the positive roots $\alpha>0$ as an indexing set for a basis of $\mathbb{R}^{N}$.

Definition 11. The FFLV polytope $P(\lambda) \subset \mathbb{R}_{\geq 0}^{N}$ for a weight $\lambda=\sum_{i=1}^{n-1} m_{i} \omega_{i} \in P^{++}$ is defined as

$$
P(\lambda)=\left\{\left(r_{\alpha}\right)_{\alpha>0} \in \mathbb{R}_{\geq 0}^{N} \mid \begin{array}{c}
\forall \mathbf{d} \in \mathscr{D}: \text { if } \beta_{0}=\alpha_{i} \text { and } \beta_{k}=\alpha_{j} \\
r_{\beta_{0}}+\cdots+r_{\beta_{k}} \leq m_{i}+\cdots+m_{j}
\end{array}\right\} .
$$

Example 3. Consider $\mathscr{F} \ell_{4}$. Then the Dyck paths are

$$
\begin{gathered}
\left(\alpha_{1}\right),\left(\alpha_{2}\right),\left(\alpha_{3}\right), \\
\left(\alpha_{1}, \alpha_{1,2}, \alpha_{2}\right),\left(\alpha_{2}, \alpha_{2,3}, \alpha_{3}\right), \\
\left(\alpha_{1}, \alpha_{1,2}, \alpha_{2}, \alpha_{2,3}, \alpha_{3}\right) \text { and }\left(\alpha_{1}, \alpha_{1,2}, \alpha_{1,3}, \alpha_{2,3}, \alpha_{3}\right)
\end{gathered}
$$

For our favorite choice of weight $\lambda=\rho=\omega_{1}+\omega_{2}+\omega_{3}$ we obtain the FFLV polytope

$$
P(\rho)=\left\{\begin{array}{l|c}
r_{\alpha_{1}} \leq 1, r_{\alpha_{2}} \leq 1, r_{\alpha_{3}} \leq 1 \\
\left(r_{\alpha}\right)_{\alpha>0} \mid \begin{array}{c}
r_{\alpha_{1}}+r_{\alpha_{1,2}}+r_{\alpha_{2}} \leq 2, r_{\alpha_{2}}+r_{\alpha_{2,3}}+r_{\alpha_{3}} \leq 2, \\
r_{\alpha_{1}}+r_{\alpha_{1,2}}+r_{\alpha_{2}}+r_{\alpha_{2,3}}+r_{\alpha_{3}} \leq 3 \\
r_{\alpha_{1}}+r_{\alpha_{1,2}}+r_{\alpha_{1,3}}+r_{\alpha_{2,3}}+r_{\alpha_{3}} \leq 3
\end{array}
\end{array}\right\} \subset \mathbb{R}_{\geq 0}^{6}
$$

The following is a corollary of [15, Proposition 11.6], which says that a strong version of the Minkowski property is satisfied by the FFLV polytope for $\mathscr{F} \ell_{n}$. It can alternatively be shown for $n=4,5$ using the methods in the proof of Proposition 2 .

Corollary 1. The FFLV polytope $P(\rho)$ satisfies the weak Minkowski property.

Remark 6. The FFLV polytope is in general not a string polytope. A computation in polymake shows that $P(\rho)$ for $\mathscr{F} \ell_{5}$ is not combinatorially equivalent to any string polytope for $\rho$. 


\section{String cones and the tropicalized flag variety}

We have seen in 2 how to obtain toric degenerations from maximal prime cones of the tropicalization of the flag varieties. We compare the different toric degenerations that arise from the different approaches. Moreover, applying [7, Lemma 3.2] we construct a weight vector from a string cone. Computational evidence for $\mathscr{F} \ell_{4}$ and $\mathscr{F} \ell_{5}$ shows that each constructed weight vector lies in the relative interior of a maximal cone in $\operatorname{trop}\left(\mathscr{F} \ell_{n}\right)$. A similar idea for a more general case is carried out in [23, §7]. For the FFLV polytope we compute weight vectors for $\mathscr{F} \ell_{n}$ with $n=4,5$ (see Example 6) following a construction given in [14].

We will now prove the result in Theorem 3 by analyzing the polytopes associated to the different toric degenerations of $\mathscr{F} \ell_{n}$ for $n=4,5$.

\begin{tabular}{|l|l|}
\hline Orbit & Combinatorially equivalent polytopes \\
\hline 1 & String 2 \\
\hline 2 & String 1 (Gelfand-Tsetlin) \\
\hline 3 & String 3 and FFLV \\
\hline 4 & - \\
\hline
\end{tabular}

Table 3 Combinatorial equivalences among the polytopes obtained from prime cones in $\operatorname{trop}\left(\mathscr{F} \ell_{4}\right)$ and string polytopes resp. the FFLV polytope.

Proof (of Theorem 3). In order to distinguish the different toric degenerations, we consider the toric varieties associated to their special fibers. In case of the degenerations induced by the string polytopes and FFLV polytope, these toric varieties are normal. This might not be true for the degenerations found in Theorem 4 and Theorem 5 . Hence, we consider two toric degenerations to be different if the normalization of their special fibers are not isomorphic.

Two toric varieties are isomorphic, if their corresponding fans are unimodular equivalent. In our case the fans are the normal fans of the polytopes. For this reason we first look for combinatorial equivalences between those. If they are not combinatorially equivalent then their normal fans can not be unimodular equivalent. We use polymake [17] for computations with polytopes.

From Table 3 one can see that for $\mathscr{F} \ell_{4}$ there is one toric degeneration, whose associated polytope is not combinatorially equivalent to any string polytope or the FFLV polytope for $\rho$. Hence, its corresponding normal toric variety is not isomorphic to any toric variety associated to these polytopes. For the toric varieties associated to the other polytopes we can not exclude isomorphism since there might be a unimodular equivalences between pairs of normal fans.

For $\mathscr{F} \ell_{5}$, Table 5 in the appendix shows that there are 168 polytopes obtained from prime cones of trop $\left(\mathscr{F} \ell_{5}\right)$ that are not combinatorially equivalent to any string polytope or the FFLV polytope for $\rho$. 
Remark 7. There are also string polytopes, which are not combinatorially equivalent to any polytope from prime cones in $\operatorname{trop}\left(\mathscr{F} \ell_{n}\right)$ for $n=4,5$. These are exactly those not satisfying MP, i.e. one string polytope for $\mathscr{F} \ell_{4}$ and 14 for $\mathscr{F} \ell_{5}$. See also Table 6 ,

From now on, we fix a reduced expression $\underline{w}_{0}=s_{i_{1}} \ldots s_{i_{N}}$ and we consider the sequence of simple roots $S=\left(\alpha_{i_{1}}, \ldots, \alpha_{i_{N}}\right)$. Recall that for a positive root $\alpha$ we denote by $f_{\alpha}$ the root vector in $\mathfrak{n}^{-} \subset \mathfrak{s l}_{n}$ of weight $-\alpha$. By [12, Lemma 2] the following holds.

Proposition 3. The universal enveloping algebra $U\left(\mathfrak{n}^{-}\right)$is linearly generated by monomials of the form $\mathbf{f}^{\mathbf{m}}=f_{\alpha_{i_{1}}}^{m_{1}} \ldots f_{\alpha_{i_{N}}}^{m_{N}}$ for $m_{i} \in \mathbb{N}$.

The proposition may be interpreted as a definition of the universal enveloping algebra. Given a weight $\lambda$, the irreducible highest weight representation $V=V(\lambda)$ is cyclically generated by a highest weight vector $v_{\lambda} \in V(\lambda)$, i.e. $V(\lambda)=U\left(\mathfrak{n}^{-}\right) \cdot v_{\lambda}$.

Example 4. For $\mathscr{F} \ell_{4}$ three root vectors in $\mathfrak{n}^{-}$are

$$
f_{\alpha_{1}}=\left[\begin{array}{llll}
0 & 0 & 0 & 0 \\
1 & 0 & 0 & 0 \\
0 & 0 & 0 & 0 \\
0 & 0 & 0 & 0
\end{array}\right], \quad f_{\alpha_{2}}=\left[\begin{array}{llll}
0 & 0 & 0 & 0 \\
0 & 0 & 0 & 0 \\
0 & 1 & 0 & 0 \\
0 & 0 & 0 & 0
\end{array}\right], \quad \text { and } \quad f_{\alpha_{3}}=\left[\begin{array}{llll}
0 & 0 & 0 & 0 \\
0 & 0 & 0 & 0 \\
0 & 0 & 0 & 0 \\
0 & 0 & 1 & 0
\end{array}\right] .
$$

Consider $V=\bigwedge^{2} \mathbb{C}^{4}$. The action of $\mathfrak{n}^{-}$on $\mathbb{C}^{4}$ is given by $f_{\alpha_{i}}\left(e_{i}\right)=e_{i+1}$ and $f_{\alpha_{i}}\left(e_{j}\right)=$ 0 for $j \neq i$. On $V$ the $\mathfrak{n}^{-}$-action is given by

$$
f_{\alpha_{i}}\left(e_{j} \wedge e_{k}\right)=f_{\alpha_{i}}\left(e_{j}\right) \wedge e_{k}+e_{j} \wedge f_{\alpha_{i}}\left(e_{k}\right) .
$$

Let $e_{1} \wedge e_{3} \in V$. Then $f_{\alpha_{2}}\left(e_{1} \wedge e_{2}\right)=e_{1} \wedge e_{3}$. In fact, $V=U\left(\mathfrak{n}^{-}\right) .\left(e_{1} \wedge e_{2}\right)$, this implies that $e_{1} \wedge e_{2}=: v_{\omega_{2}}$ is a highest weight vector. If we fix $\underline{w}_{0}=s_{1} s_{2} s_{1} s_{3} s_{2} s_{1}$, we have $U\left(\mathfrak{n}^{-}\right)=\left\langle f_{\alpha_{1}}^{m_{1}} f_{\alpha_{2}}^{m_{2}} f_{\alpha_{1}}^{m_{3}} f_{\alpha_{3}}^{m_{4}} f_{\alpha_{2}}^{m_{5}} f_{\alpha_{1}}^{m_{6}}: m_{i} \in \mathbb{N}\right\rangle$. Hence

$$
\mathbf{f}^{(0,1,0,0,0,0)}\left(e_{1} \wedge e_{2}\right)=\mathbf{f}^{(0,0,0,0,0,1,0)}\left(e_{1} \wedge e_{2}\right) .
$$

As seen in Example 4 the monomial $\mathbf{f}^{\mathrm{m}}$ for a given weight vector $v \in V$ with $\mathbf{f}^{\mathbf{m}}\left(v_{\lambda}\right)=v$ is not unique. To fix this, we define a term order on the monomials $\mathbf{f}^{\mathbf{m}}$ generating $U\left(\mathfrak{n}^{-}\right)$and pick the minimal monomial with this property. We fix for $\mathbf{m}, \mathbf{n} \in \mathbb{N}^{N}$ the order

$$
\mathbf{f}^{\mathbf{m}} \succ \mathbf{f}^{\mathbf{n}}, \text { if } \operatorname{deg}\left(\mathbf{f}^{\mathbf{m}}\right)>\operatorname{deg}\left(\mathbf{f}^{\mathbf{n}}\right) \text { or } \operatorname{deg}\left(\mathbf{f}^{\mathbf{m}}\right)=\operatorname{deg}\left(\mathbf{f}^{\mathbf{n}}\right) \text { and } \mathbf{m}<_{l e x} \mathbf{n} .
$$

The connection to trop $\left(\mathscr{F} \ell_{n}\right)$ is established through Plücker coordinates. For $J=\left\{j_{1}, \ldots, j_{k}\right\} \subset[n], p_{J}$ is given by the Plücker embedding as $\left(e_{j_{1}} \wedge \ldots \wedge e_{j_{k}}\right)^{*} \in$ $\left(\wedge^{k} \mathbb{C}^{n}\right)^{*}$, the dual vector space. Now $\wedge^{k} \mathbb{C}^{n}$ is the fundamental representation $V\left(\omega_{k}\right)=U\left(\mathfrak{n}^{-}\right) \cdot\left(e_{1} \wedge \ldots \wedge e_{k}\right)$ (see Example 4). Denote by $\mathbf{m}_{J}$ the unique multiexponent such that $\mathbf{f}^{\mathbf{m}_{J}}$ is $\prec$-minimal satisfying $\mathbf{f}^{\mathbf{m}}\left(e_{1} \wedge \ldots \wedge e_{k}\right)=e_{j_{1}} \wedge \ldots \wedge e_{j_{k}}$.

Following a construction given in [7, Proof of Lemma 3.2], we define the linear form $e: \mathbb{N}^{N} \rightarrow \mathbb{N}$ as $e(\mathbf{m})=2^{N-1} m_{1}+2^{N-2} m_{2}+\ldots+2 m_{N-1}+m_{N}$. This is a particular choice satisfying $\mathbf{m} \succ \mathbf{n} \Rightarrow e(\mathbf{m})>e(\mathbf{n})$ for $\mathbf{m}, \mathbf{n} \in \mathbb{N}^{N}$. 
Definition 12. For a fixed reduced expression $\underline{w}_{0}$ the weight of the Plücker variable $p_{J}$ is $e\left(\mathbf{m}_{J}\right)$. We fix the weight vector $\mathbf{w}_{\underline{w}_{0}}$ in $\mathbb{R}^{\left(\begin{array}{l}n \\ 1\end{array}\right)+\left(\begin{array}{l}n \\ 2\end{array}\right)+\cdots+\left(\begin{array}{c}n \\ n-1\end{array}\right)}$ to be

$$
\mathbf{w}_{\underline{w}_{0}}=\left(e\left(\mathbf{m}_{1}\right), e\left(\mathbf{m}_{2}\right), \ldots, e\left(\mathbf{m}_{2,3, \ldots, n}\right)\right) .
$$

Example 5. We continue as in Example 4 with the fixed reduced expression $\underline{w}_{0}=$ $s_{1} s_{2} s_{1} s_{3} s_{2} s_{1}$ for $\mathscr{F} \ell_{4}$. The Plücker coordinate $p_{13}$ in $\mathscr{F} \ell_{4}$ is $\left(e_{1} \wedge e_{3}\right)^{*}$. The corresponding minimal monomial among those satisfying $\mathbf{f}^{\mathbf{m}}\left(e_{1} \wedge e_{2}\right)=e_{1} \wedge e_{3}$ is $\mathbf{f}^{(0,1,0,0,0,0)}$. Hence the weight of $p_{13}$ is $e(0,1,0,0,0,0)=1 \cdot 2^{4}=16$. Similarly, we obtain the weights of all Plücker coordinates and

$$
\mathbf{w}_{\underline{w}_{0}}=(0,32,24,7,0,16,6,48,38,30,0,4,20,52) .
$$

Table 2 contains all weight vectors for $\mathscr{F} \ell_{4}$ constructed in the way just described.

Proposition 4. Consider $\mathscr{F} \ell_{n}$ with $n=4,5$. The above construction produces a weight vector $\mathbf{w}_{\underline{w}_{0}}$ for every string cone. This weight vector lies in the relative interior of a maximal cone of trop $\left(\mathscr{F} \ell_{n}\right)$. If further the string cone satisfies MP, then $\mathbf{w}_{\underline{w}_{0}}$ lies in the relative interior of a prime cone whose associated polytope is combinatorially equivalent to $Q_{\underline{w}_{0}}(\rho)$.

Proof. The constructed weight vectors $\mathbf{w}_{\underline{w}_{0}}$ can be found in Table 2 for $\mathscr{F} \ell_{4}$ and Table 6 in the appendix for $\mathscr{F} \ell_{5}$. A computation in Macaulay 2 shows that all initial ideals $\operatorname{in}_{\mathbf{w}_{\underline{w}_{0}}}\left(I_{n}\right)$ for $n=4,5$ are binomial, hence in the relative interiors of maximal cones of trop $\left(\mathscr{F} \ell_{n}\right)$.

Moreover, if MP is satisfied we check using polymake that the polytope constructed from the maximal prime cone $C_{\underline{w}_{0}}$ with $\mathbf{w}_{\underline{w}_{0}}$ in its relative interior is combinatorially equivalent to the string polytope $Q_{\underline{w}}(\bar{\rho})$. See Table 2 and Table 6

Computational evidence leads us to the following conjecture.

Conjecture 1. Let $n \geq 3$ be an arbitrary integer. For every reduced expression $\underline{w}_{0}$, the weight vector $\mathbf{w}_{\underline{w}_{0}}$ lies in the relative interior of a maximal cone in trop $\left(\mathscr{F} \ell_{n}\right)$.

In particular, if the string cone satisfies MP this vector lies in the relative interior of the prime cone $C$, whose associated polytope is combinatorially equivalent to the string polytope $Q_{\underline{w}_{0}}(\rho)$.

The following example discusses a similar construction of weight vectors for the FFLV polytope.

Example 6. Consider for $\mathscr{F} \ell_{4}$ the sequence of positive roots

$$
S=\left(\alpha_{1}+\alpha_{2}+\alpha_{3}, \alpha_{1}+\alpha_{2}, \alpha_{2}+\alpha_{3}, \alpha_{1}, \alpha_{2}, \alpha_{3}\right) .
$$

By [12, Example 1], Proposition 3 is also true for this choice of $S$. More generally speaking, Proposition 3 holds for every sequence containing all positive roots ordered by height. The height of a positive root is the number of simple summands. Such sequences are called $P B W$-sequences with good ordering in [12]. 
With this choice of $S$ we apply the aformentioned procedure to obtain a unique multi-exponent $\mathbf{m}_{J}$ for each Plücker variable $p_{J}$. Taking the convex hull of all multiexponents $\mathbf{m}_{J}$ for $J \subset\{1, \ldots, 4\}$ yields the FFLV polytope from Definition 11 with respect to the embedding $\mathscr{F} \ell_{4} \hookrightarrow \mathbb{P}(V(\rho))$. Then we define linear forms

$$
\begin{gathered}
e^{\min }\left(\mathbf{m}_{J}\right)=m_{1}+2 m_{2}+m_{3}+2 m_{4}+m_{5}+m_{6}, \\
e^{\mathrm{reg}}\left(\mathbf{m}_{J}\right)=3 m_{1}+4 m_{2}+2 m_{3}+3 m_{4}+2 m_{5}+m_{6},
\end{gathered}
$$

according to the degrees defined in [14]. We obtain in analogy to Definition 12] the corresponding weight vectors

$$
\begin{aligned}
\mathbf{w}^{\text {min }} & =(0,2,2,1,0,1,1,2,1,2,0,1,1,1), \\
\mathbf{w}^{\text {reg }} & =(0,3,4,3,0,2,2,4,3,5,0,1,2,3) .
\end{aligned}
$$

A computation in Macaulay2 shows that $\operatorname{in}_{\mathbf{w}^{\min }}\left(I_{4}\right)=\mathrm{in}_{\mathbf{w}^{\mathrm{reg}}}\left(I_{4}\right)$ is a binomial prime ideal. Hence $\mathbf{w}^{\mathrm{min}}$ and $\mathbf{w}^{\text {reg }}$ lie in the relative interior of the same prime cone $C \subset \operatorname{trop}\left(\mathscr{F} l_{4}\right)$. Using polymake [17] we verify that the polytope associated to $C$ is combinatorially equivalent to the FFLV polytope $P(\rho)$. We did the analogue of this computation for $\mathscr{F} \ell_{5}$ and the outcome is the same, $\operatorname{in}_{\mathbf{w}^{\min }}\left(I_{5}\right)=\mathrm{in}_{\mathbf{w}^{\text {reg }}}\left(I_{5}\right)=\mathrm{in}_{C}\left(I_{5}\right)$ with the polytope associated to $C$ being combinatorially equivalent to $P(\rho)$. The weight vectors $\mathbf{w}^{\text {min }}$ and $\mathbf{w}^{\text {reg }}$ for $\mathscr{F} \ell_{5}$ can be found in Table 6 in the appendix.

\section{Toric degenerations from non-prime cones}

As we have seen in $\$ 3$, not all maximal cones in the tropicalization of a variety give rise to prime initial ideals and hence to toric degenerations. In fact, there may also be tropicalizations without prime cones (see Example 7). Let $X$ be a subvariety of a toric variety $Y$. In this section, we give a recursive procedure (Procedure 1) to compute a new embedding $X^{\prime}$ of $X$ in case $\operatorname{trop}(X)$ has non-prime cones. Let $C$ be a non-prime cone. If the algorithm terminates, the new variety $X^{\prime}$ has more prime cones than $\operatorname{trop}(X)$ and at least one of them is projecting onto $C$. We apply this procedure to $\mathscr{F} \ell_{4}$ and compare the new toric degenerations with those obtained so far (see Proposition 5). The procedure terminates for $\mathscr{F} \ell_{4}$, but we are still investigating the conditions for which this is true in general.

We now explain Procedure 1. Consider a toric variety $Y$ whose total coordinate ring is $\mathbb{C}\left[x_{0}, \ldots, x_{n}\right]$ with associated $\mathbb{Z}^{k}$-degree deg : $\mathbb{Z}^{n+1} \rightarrow \mathbb{Z}^{k}$. Let $X$ be the subvariety of $Y$ associated to an ideal $I \subset \mathbb{C}\left[x_{0}, \ldots, x_{n}\right]$, where the Krull dimension of $A=\mathbb{C}\left[x_{0}, \ldots, x_{n}\right] / I$ is $d$. Denote by $\operatorname{trop}(V(I))$ the tropicalization of $X$ intersected with the torus of $Y$. Suppose there is a non-prime cone $C \subset \operatorname{trop}(V(I))$ with multiplicity one. By Lemma2, we have that $I\left(W_{C}\right)$ is the unique toric ideal in the primary decomposition of $\operatorname{in}_{C}(I)$, hence $\operatorname{in}_{C}(I) \subset I\left(W_{C}\right)$. We can compute $I\left(W_{C}\right)$ using the function primaryDecomposition in Macaulay2. Fix a minimal binomial generating set $G$ of $I\left(W_{C}\right)$, and let $L_{C}=\left\{f_{1}, \ldots, f_{s}\right\}$ be the set consisting of binomials 
Procedure 1: Computing new embeddings of the variety $X$ in case $\operatorname{trop}(X)$ contains non-prime cones

Input: $A=\mathbb{C}\left[x_{0}, \ldots, x_{n}\right] / I$, where $\mathbb{C}\left[x_{0}, \ldots, x_{n}\right]$ is the total coordinate ring of the toric variety $Y$ and $I$ defines the subvariety $V(I) \subset Y, C$ a non-prime cone of $\operatorname{trop}(V(I))$.

\section{Initialization:}

Compute the primary decomposition of $\operatorname{in}_{C}(I)$;

$I\left(W_{C}\right)=$ unique prime toric component in the decomposition;

$G=$ minimal generating set of $I\left(W_{C}\right)$.

Compute a list of binomials $L_{C}=\left\{f_{1}, \ldots, f_{s}\right\}$ in $G$, which are not in $\operatorname{in}_{C}(I)$;

$A^{\prime}=\mathbb{C}\left[x_{0}, \ldots, x_{n}, y_{1}, \ldots, y_{s}\right] / I^{\prime}$ with $I^{\prime}=I+\left\langle y_{1}-f_{1}, \ldots, y_{s}-f_{s}\right\rangle$;

$V\left(I^{\prime}\right)$ subvariety of $Y^{\prime}$ whose total coordinate ring is $\mathbb{C}[Y]:=\mathbb{C}\left[x_{0}, \ldots, x_{n}, y_{1}, \ldots, y_{s}\right]$.

Compute trop $\left(V\left(I^{\prime}\right)\right)$;

for all prime cones $C^{\prime} \in \operatorname{trop}\left(V\left(I^{\prime}\right)\right)$ do

if $\pi\left(C^{\prime}\right)$ is contained in the relative interior of $C$ then

$\mathrm{L}$ Output: The algebra $A^{\prime}$ and the ideal $\operatorname{in}_{C^{\prime}}\left(I^{\prime}\right)$ of a toric degeneration of $V\left(I^{\prime}\right)$. else

$\mathrm{L}$ Apply the procedure again to $A^{\prime}$ and $C^{\prime}$.

in $G$, which are not in $\operatorname{in}_{C}(I)$. By Hilbert's Basis Theorem, $s$ is a finite number. The absence of these binomials in $\operatorname{in}_{C}(I)$ is the reason why the initial ideal is not equal to $I\left(W_{C}\right)$. We introduce new variables $\left\{y_{1}, \ldots, y_{s}\right\}$ and consider the algebra $A^{\prime}=\mathbb{C}\left[x_{0}, \ldots, x_{n}, y_{1}, \ldots, y_{s}\right] / I^{\prime}$, where

$$
I^{\prime}=I+\left\langle y_{1}-f_{1}, \ldots, y_{s}-f_{s}\right\rangle
$$

The ideal $I^{\prime}$ is a homogeneous ideal in $\mathbb{C}\left[x_{0}, \ldots, x_{n}, y_{1}, \ldots, y_{s}\right]$ graded by

$$
\left(\operatorname{deg}\left(x_{0}\right), \ldots, \operatorname{deg}\left(x_{n}\right), \operatorname{deg}\left(f_{1}\right), \ldots, \operatorname{deg}\left(f_{s}\right)\right) .
$$

The new variety $V\left(I^{\prime}\right)$ is a subvariety of a toric variety $Y^{\prime}$, which has total coordinate $\mathbb{C}\left[Y^{\prime}\right]:=\mathbb{C}\left[x_{0}, \ldots, x_{n}, y_{1}, \ldots, y_{s}\right]$. For example, if $V(I)$ is a subvariety of a projective space then $V\left(I^{\prime}\right)$ is contained in a weighted projective space.

Since the algebras $A$ and $A^{\prime}$ are isomorphic as graded algebras, the varieties $V(I)$ and $V\left(I^{\prime}\right)$ are isomorphic. We have a monomial map

$$
\pi: \mathbb{C}\left[x_{0}, \ldots, x_{n}\right] / I \rightarrow \mathbb{C}\left[x_{0}, \ldots, x_{n}, y_{1}, \ldots, y_{s}\right] / I^{\prime}
$$

inducing a surjective map $\operatorname{trop}(\pi): \operatorname{trop}\left(V\left(I^{\prime}\right)\right) \rightarrow \operatorname{trop}(V(I))$ (see [25. Corollary 3.2.13]). The map trop $(\pi)$ is the projection onto the first $n$ coordinates. Suppose there exists a prime cone $C^{\prime} \subset \operatorname{trop}\left(V\left(I^{\prime}\right)\right)$, whose projection has a non-empty intersection with the relative interior of $C$. Then by construction we have $\operatorname{in}_{C}(I) \subset$ $\operatorname{in}_{C^{\prime}}\left(I^{\prime}\right) \cap \mathbb{C}\left[x_{0}, \ldots, x_{n}\right]$ and the procedure terminates. In this way we obtain a new initial ideal $\operatorname{in}_{C^{\prime}}\left(I^{\prime}\right)$ which is toric and hence gives a new toric degeneration of the variety $V\left(I^{\prime}\right) \cong V(I)$. If only non-prime cones are projecting to $C$ then run this procedure again with $A^{\prime}$ and $C^{\prime}$, where the latter is a maximal cone of $\operatorname{trop}\left(V\left(I^{\prime}\right)\right)$, which 
projects to $C$. We can then repeat the procedure starting from a different non-prime cone.

The function to apply Procedure 1 is findNewToricDegenerations and it is part of the package ToricDegenerations. This will compute only one reembedding for each non-prime cone. It is possible to use mapMaximalCones to obtain the image of trop $\left(V\left(I^{\prime}\right)\right)$ under the map $\pi$.

Remark 8. If $f_{i}$ is a polynomial in $\mathbb{k}\left[x_{0}, x_{1}, \ldots, x_{n}\right]$ with the standard grading and $\operatorname{deg}\left(f_{i}\right)>1$, then we need to homogenize the ideal $I^{\prime}$ before computing the tropicalization with Gfan. This is done by adding a new variable $h$. The homogenization of $I^{\prime}$ with respect to $h$ is denoted by $I_{\text {proj } j}^{\prime} \subseteq \mathbb{k}\left[x_{0}, \ldots, x_{n}, y_{1}, \ldots, y_{s}, h\right]$. Then by [25, Proposition 2.6.1] for every $\mathbf{w}$ in $\mathbb{R}^{n+s+2}$ the ideal $\operatorname{in}_{\mathbf{w}}\left(I^{\prime}\right)$ is obtained from $\operatorname{in}_{(\mathbf{w}, 0)}\left(I_{\text {proj }}^{\prime}\right)$ by setting $h=1$.

If the cone $C$ is prime, we can construct a valuation $\mathfrak{v}_{C}$ on $\mathbb{k}\left[x_{0}, \ldots, x_{n}\right] / I$ in the following way. Consider the matrix $W_{C}$ in Equation (1). For monomials $m_{i}=c \mathbf{x}^{\alpha_{i}} \in$ $\mathbb{k}\left[x_{0}, \ldots x_{n}\right]$ define

$$
\mathfrak{v}\left(m_{i}\right)=W_{C} \alpha_{i} \quad \text { and } \quad \mathfrak{v}\left(\sum_{i} m_{i}\right)=\min _{i}\left\{\mathfrak{v}\left(m_{i}\right)\right\},
$$

where the minimum on the right side is taken with respect to the lexicographic order on $\left(\mathbb{Z}^{d},+\right)$. This is a valuation on $\mathbb{k}\left[x_{0}, \ldots, x_{n}\right]$ of rank equal to the Krull dimension of $A$ for every cone $C$. Composing $\mathfrak{v}$ with the quotient morphism $p: \mathbb{k}\left[x_{0}, \ldots, x_{n}\right] \rightarrow$ $\mathbb{k}\left[x_{0}, \ldots, x_{n}\right] / I$ we obtain a map $\mathfrak{v}_{C}$, which is a valuation if and only if the cone $C$ is prime. Moreover, in [23] Kaveh and Manon prove that a cone $C$ in $\operatorname{trop}(V(I))$ is prime if and only if $A=\mathbb{k}\left[x_{0}, \ldots, x_{n}\right] / I$ has a finite Khovanskii basis for the valuation $\mathfrak{v}_{C}$ constructed from the cone $C$. Recall that a Khovanskii basis for an algebra $A$ with valuation $\mathfrak{v}_{C}$ is a subset $B$ of $A$ such that $\mathfrak{v}_{C}(B)$ generates the value semigroup $S\left(A, \mathfrak{v}_{C}\right)=\left\{\mathfrak{v}_{C}(f): f \in A \backslash\{0\}\right\}$.

Procedure 1 can be interpreted as finding an extension $\mathfrak{v}_{C^{\prime}}$ of $\mathfrak{v}_{C}$ so that $A^{\prime}$ has finite Khovanskii basis with respect to $\mathfrak{v}_{C^{\prime}}$. The Khovanskii basis is given by the images of $x_{0}, \ldots, x_{n}, y_{1}, \ldots, y_{s}$ in $A^{\prime}$. We illustrate the procedure in the following example.

Example 7. Consider the algebra $A=\mathbb{C}[x, y, z] /\langle x y+x z+y z\rangle$. The tropicalization of $V(\langle x y+x z+y z\rangle) \subset \mathbb{P}^{2}$ has three maximal cones. The corresponding initial ideals are $\langle x z+y z\rangle,\langle x y+y z\rangle$ and $\langle x y+x z\rangle$, none of which is prime. Hence they do not give rise to toric degenerations. The matrices associated to each cone are

$$
W_{C_{1}}=\left[\begin{array}{ccc}
0 & 0 & -1 \\
1 & 1 & 1
\end{array}\right], \quad W_{C_{2}}=\left[\begin{array}{ccc}
0 & -1 & 0 \\
1 & 1 & 1
\end{array}\right] \quad \text { and } \quad W_{C_{3}}=\left[\begin{array}{rrr}
-1 & 0 & 0 \\
1 & 1 & 1
\end{array}\right] .
$$

We now apply Procedure 1 to the cone $C_{1}$. The initial ideal associated to $C_{1}$ is generated by $x z+y z$. In this case $\operatorname{in}_{C_{1}}(I)=\langle z\rangle \cdot\langle x+y\rangle$ hence for the missing binomial $x+y$ we adjoin a new variable $u$ to $\mathbb{C}[x, y, z]$ and the new relation $u-x-y$ to $I$. We have 


$$
I^{\prime}=\langle x y+x z+y z, u-x-y\rangle \text { and } A^{\prime}=\mathbb{C}[x, y, z, u] / I^{\prime}
$$

with $V\left(I^{\prime}\right)$ a subvariety of $\mathbb{P}^{3}$. After computing the tropicalization of $V\left(I^{\prime}\right)$ we see that there exists a prime cone $C^{\prime}$ such that $\pi\left(C^{\prime}\right)=C$. The matrix $W_{C^{\prime}}$ associated to the cone $C^{\prime}$ is

$$
W^{\prime}=\left[\begin{array}{rrrr}
0 & 0 & -1 & 1 \\
1 & 1 & 1 & 1
\end{array}\right] \text {. }
$$

The initial ideal $\operatorname{in}_{C^{\prime}}\left(I^{\prime}\right)$ gives a toric degeneration of $V\left(I^{\prime}\right)$. The image of the set $\{x, y, z, u\}$ in $A^{\prime}$ is a Khovanskii basis for $S\left(A^{\prime}, \mathfrak{v}_{C^{\prime}}\right)$. Repeating this process for the cones $C_{2}$ and $C_{3}$ of trop $(V(x y+x z+y z))$, we get prime cones $C_{2}^{\prime}$ and $C_{3}^{\prime}$ whose projections are $C_{2}$ and $C_{3}$ respectively. Hence there is a valuation with finite Khovanskii basis and a corresponding toric degeneration for every maximal cone.

We now apply Procedure 1 to trop $\left(\mathscr{F} \ell_{4}\right)$.

Proposition 5. Each of the non-prime cones of trop $\left(\mathscr{F} \ell_{4}\right)$ gives rise to three toric degenerations, which are not isomorphic to any degeneration coming from the prime cones of trop $\left(\mathscr{F} \ell_{4}\right)$. Moreover, two of the three new polytopes are combinatorially equivalent to the previously missing string polytopes for $\rho$ in the class String 4.

Proof. By Theorem 4 we know that trop $\left(\mathscr{F} \ell_{4}\right)$ has six non-prime cones forming one $S_{4} \rtimes \mathbb{Z}_{2}$-orbit. Hence, we apply Procedure 1 to only one non-prime cone. The result for the other non-prime cones will be the same up to symmetry. In particular, the obtained toric degenerations from one cone will be isomorphic to those coming from another cone. We describe the results for the maximal cone $C$ associated to the initial ideal $\operatorname{in}_{C}\left(I_{4}\right)$ defined by the following binomials:

$$
\begin{aligned}
& p_{4} p_{123}-p_{3} p_{124}, p_{24} p_{134}-p_{14} p_{234}, p_{23} p_{134}-p_{13} p_{234}, p_{2} p_{14}-p_{1} p_{24}, \\
& p_{2} p_{13}-p_{1} p_{23}, \quad p_{24} p_{123}-p_{23} p_{124}, p_{14} p_{123}-p_{13} p_{124}, p_{4} p_{23}-p_{3} p_{24} \\
& p_{4} p_{13}-p_{3} p_{14} \text {, and } p_{14} p_{23}-p_{13} p_{24} \text {. }
\end{aligned}
$$

We define the ideal $I^{\prime}=I_{4}+\left\langle w-p_{2} p_{134}+p_{1} p_{234}\right\rangle$. The grading on the variables $p_{1}, \ldots, p_{234}$ and $w$ is given by the matrix

$$
\left[\begin{array}{lllllllllllllll}
1 & 1 & 1 & 1 & 0 & 0 & 0 & 0 & 0 & 0 & 0 & 0 & 0 & 0 & 1 \\
0 & 0 & 0 & 0 & 1 & 1 & 1 & 1 & 1 & 1 & 0 & 0 & 0 & 0 & 0 \\
0 & 0 & 0 & 0 & 0 & 0 & 0 & 0 & 0 & 0 & 1 & 1 & 1 & 1 & 1
\end{array}\right] .
$$

It extends the grading on the variables $p_{1}, \ldots, p_{234}$ given by the matrix $D$ in (2). The tropical variety trop $\left(V\left(I^{\prime}\right)\right)$ has 105 maximal cones, 99 of which are prime. Among them we can find three maximal prime cones, which are mapped to $C$ by trop $(\pi)$ (see Figure 6). We compute the polytopes associated to the normalization of these three toric degenerations by applying the same methods as in Theorem 4, Using polymake we check that two of them are combinatorially equivalent to the string polytopes for $\rho$ in the class String 4 . Moreover, none of them is combinatorially equivalent to any polytope coming from prime cones of trop $\left(\mathscr{F} \ell_{4}\right)$, hence they define different toric degenerations. 
$\operatorname{trop}\left(V\left(I^{\prime}\right)\right) \supset C_{1}, C_{2}, C_{3}$

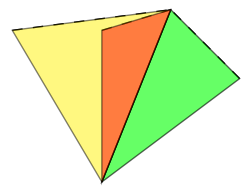

$\downarrow \operatorname{trop}(\pi)$

$\operatorname{trop}\left(\mathscr{F} \ell_{4}\right) \supset C$

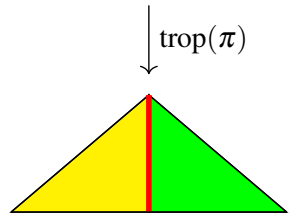

Fig. 6 The three triangles above represent the three cones in $\operatorname{trop}\left(V\left(I^{\prime}\right)\right)$ which project down to the non-prime cone $C$ in trop $\left(\mathscr{F} \ell_{4}\right)$.

Remark 9. Procedure 1 could be applied also to $\mathscr{F} \ell_{5}$ but we have not been able to do so. In fact, the tropicalization for trop $\left(V\left(I_{5}^{\prime}\right)\right)$ did not terminate since the computation can not be simplified by symmetries.

Acknowledgements This article was initiated during the Apprenticeship Weeks (22 August-2 September 2016), led by Bernd Sturmfels, as part of the Combinatorial Algebraic Geometry Semester at the Fields Institute. The authors are grateful to the Max Planck Institute MiS Leipzig, where part of this project was carried out. We are grateful to Diane Maclagan, Kiumars Kaveh, and Kristin Shaw for inspiring conversations. We also would like to thank Diane Maclagan, Yue Ren and five anonymous referees for their comments on an earlier version of this manuscript. Further, L.B. and F.M. would like to thank Ghislain Fourier and Xin Fang for many inspiring discussions. K.M would like to express her gratitude to Dániel Joó for many helpful conversations. F.M. was supported by a postdoctoral fellowship from the Einstein Foundation Berlin. S.L. was supported by EPSRC grant 1499803.

\section{References}

1. Valery Alexeev and Michel Brion. Toric degenerations of spherical varieties. Selecta Mathematica, 10(4):453-478, 2005.

2. Dave Anderson. Okounkov bodies and toric degenerations. Mathematische Annalen, 356(3):1183-1202, 2013.

3. Arkady Berenstein and Andrei Zelevinsky. Tensor product multiplicities, canonical bases and totally positive varieties. Inventiones mathematicae, 143(1):77-128, 2001.

4. René Birkner. Polyhedra.m2 - A Macaulay2 package for computations with convex polyhedra, cones, and fans.

5. Lara Bossinger, Xin Fang, Fourier Ghislain, Milena Hering, and Martina Lanini. Toric degenerations of $\operatorname{Gr}(2, n)$ and $\operatorname{Gr}(3,6)$ via plabic graphs. arXiv preprint arXiv:1612.03838, 2016.

6. Lara Bossinger and Ghislain Fourier. String cone and superpotential combinatorics for flag and schubert varieties in type A. arXiv preprint arXiv:1611.06504.

7. Philippe Caldero. Toric degenerations of Schubert varieties. Transformation groups, 7(1):5160, 2002. 
8. David Cox, John Little, and Donal O'Shea. Ideals, varieties, and algorithms. Undergraduate Texts in Mathematics. Springer-Verlag, New York, 1992. An introduction to computational algebraic geometry and commutative algebra.

9. David A. Cox, John B. Little, and Henry K. Schenck. Toric varieties. American Mathematical Soc., 2011.

10. Wolfram Decker, Gert-Martin Greuel, Gerhard Pfister, and Hans Schönemann. Singular 4-1-0 — A computer algebra system for polynomial computations. http://www.singular.uni-kl.de 2016.

11. David Eisenbud. Commutative Algebra: with a view toward algebraic geometry, volume 150. Springer Science \& Business Media, 2013.

12. Xin Fang, Ghislain Fourier, and Peter Littelmann. Essential bases and toric degenerations arising from birational sequences. Adv. Math., 312:107-149, 2017.

13. Xin Fang, Ghislain Fourier, and Peter Littelmann. On toric degenerations of flag varieties. In Representation theory-current trends and perspectives, EMS Ser. Congr. Rep., pages 187232. Eur. Math. Soc., Zürich, 2017.

14. Xin Fang, Ghislain Fourier, and Markus Reineke. PBW-type filtration on quantum groups of type $A_{n}$. Journal of Algebra, 449:321-345, 2016.

15. Evgeny Feigin, Ghislain Fourier, and Peter Littelmann. PBW filtration and bases for irreducible modules in type $A_{n}$. Transformation Groups, 16(1):71-89, 2011.

16. Evgeny Feigin, Ghislain Fourier, and Peter Littelmann. Favourable modules: filtrations, polytopes, Newton-Okounkov bodies and flat degenerations. Transform. Groups, 22(2):321-352, 2017.

17. Ewgenij Gawrilow and Michael Joswig. Polymake: a framework for analyzing convex polytopes. In Polytopes-combinatorics and computation, pages 43-73. Springer, 2000.

18. Oleg Gleizer and Alexander Postnikov. Littlewood-Richardson coefficients via Yang-Baxter equation. International Mathematics Research Notices, 2000(14):741-774, 2000.

19. Daniel R. Grayson and Michael E. Stillman. Macaulay2, a software system for research in algebraic geometry. Available at http://www.math.uiuc.edu/Macaulay2/

20. James E. Humphreys. Introduction to Lie algebras and representation theory, volume 9 of Springer Science \& Business Media. 1972.

21. Anders N. Jensen. Gfan, a software system for Gröbner fans and tropical varieties. Available at http://home.imf.au.dk/jensen/software/gfan/gfan.html

22. Anders N. Jensen. Gfan version 0.5: A users manual. Available at http://home.math.au.dk/jensen/software/gfan/gfanmanual0.5.pdf

23. Kiumars Kaveh and Christopher Manon. Khovanskii bases, higher rank valuations and tropical geometrypolytopes and tropical geometry of projective varieties". arXiv preprint arXiv:1610.00298, 2016.

24. Peter Littelmann. Cones, crystals, and patterns. Transformation groups, 3(2):145-179, 1998.

25. Diane Maclagan and Bernd Sturmfels. Introduction to tropical geometry, volume 161 of American Mathematical Soc. 2015.

26. Ezra Miller and Bernd Sturmfels. Combinatorial commutative algebra, volume 227 of Graduate Texts in Mathematics. Springer-Verlag, New York, 2005.

27. Fatemeh Mohammadi and Kristin Shaw. Toric degenerations of Grassmannians from matching fields. In preparation, 2016.

28. Gerhard Pfister, Wolfram Decker, Hans Schoenemann, and Santiago Laplagne. Primdec.lib.A Singular 4-1-0 library for computing the primary decomposition and radical of ideals.

29. David Speyer and Bernd Sturmfels. The tropical Grassmannian. Advances in Geometry, 4(3):389-411, 2004.

30. Bernd Sturmfels. Fitness, apprenticeship, and polynomials. arXiv preprint arXiv:1612.03539.

31. Bernd Sturmfels. Gröbner bases and convex polytopes, volume 8 of American Mathematical Soc. 1996.

32. Jakub Witaszek. The degeneration of the Grassmannian into a toric variety and the calculation of the eigenspaces of a torus action. Journal of Algebraic Statistics, 6(1), 2015. 


\section{Appendix}

In this Appendix we provide numerical evidence of our computations. Table 4 contains data on the non-prime maximal cones of trop $\left(\mathscr{F} \ell_{5}\right)$. In Table 5 there is information on the polytopes obtained from maximal prime cones of $\operatorname{trop}\left(\mathscr{F} \ell_{5}\right)$. This includes the F-vectors, combinatiral equivalences among the polytopes, and between those and the string polytopes, resp. FFLV polytope, for $\rho$. Lastly Table 6 contains information on the string polytopes and FFLV polytope for $\mathscr{F} \ell_{5}$, such as the weight vectors constructed in $\$ 5$ primeness of the initial ideals with respect to these vectors, and the MP property.

\section{Algebraic and combinatorial invariants of trop $\left(\mathscr{F} \ell_{5}\right)$}

Below we collect in a table all the information about the non-prime initial ideals of $\mathscr{F} \ell_{5}$ up to symmetry.

\begin{tabular}{|l|l|}
\hline Number of Orbits & \#Generators \\
\hline 30 & 69 \\
267 & 66 \\
37 & 68 \\
11 & 70 \\
10 & 71 \\
2 & 73 \\
\hline
\end{tabular}

Table 4 Data for non-prime initial ideals of $\mathscr{F} l_{5}$.

The following table shows the F-vectors of the polytopes associated to maximal prime cones of $\operatorname{trop}\left(\mathscr{F} \ell_{5}\right)$ for one representative in each orbit. The last column contains information on the existence of a combinatorial equivalence between these polytopes and the string polytopes resp. FFLV polytope for $\rho$. The initial ideals are all Cohen-Macaulay.

\begin{tabular}{|l|l|l|}
\hline Orbit & F-vector & Combinatorial equivalences \\
\hline 0 & 475295684171424115690116435820189937437 & \\
\hline 1 & 456279978431302314038101594938156530130 & \\
\hline 2 & 42525737108116261233387794201131625326 & \\
\hline 3 & 3932313620098331012570213297102720122 & \\
\hline 4 & 43326217230117961247388474219131825326 & \\
\hline 5 & 43526307246118101247988484219131825326 & \\
\hline 6 & 42525536988113171188883883987124524025 & \\
\hline 7 & 45027517677126991364898634800152929730 & \\
\hline
\end{tabular}




\begin{tabular}{|l|l|l|}
\hline Orbit & -vector & Combinatorial equivalences \\
\hline 8 & 43526307246118101247988484219131825326 & \\
\hline 9 & 41925226922112431184283733985124524025 & \\
\hline 10 & 453278578171299914027101574938156530130 & \\
\hline 11 & 463288582371398715474115325788189537437 & \\
\hline 12 & 463285280201336514459105015121162731331 & \\
\hline 13 & 457284080781363814954109965413172633032 & \\
\hline 14 & 454281980161354014870109685427174433733 & \\
\hline 15 & 445274877701305014254104645161165832232 & \\
\hline 16 & 44126817438122281305693694525143027628 & \\
\hline 17 & 440270476021268413752100144897156030130 & \\
\hline 18 & 471292382981399515369113695667184536336 & \\
\hline 19 & 464288382001386115258113135651184336336 & \\
\hline 20 & 467291183091409715574115865804189737437 & \\
\hline 21 & 461287682251399315509115755814190337537 & \\
\hline 22 & 39723636416103131075575363561110921523 & \\
\hline 23 & 43726697447123191323695564642147528629 & \\
\hline 24 & 42525536988113171188883883987124524025 & \\
\hline 25 & 41524986861111581177283393976124424025 & \\
\hline 26 & 470294284361437715944118895955193937937 & \\
\hline 27 & 460285681091365614929109445374171232832 & \\
\hline 28 & 44927417634125941348797024695148628729 & \\
\hline 29 & 42725927181117781252389264270133425526 & \\
\hline 30 & 42525737108116261233387794201131625326 & FFLV \\
\hline 31 & 44327087557124951341196674686148528729 & \\
\hline 32 & 39723636416103131075575363561110921523 & S22 \\
\hline 33 & 42525536988113171188883883987124524025 & \\
\hline 34 & 41925226922112431184283733985124524025 & \\
\hline 35 & 40524076518104421085175783571111021523 & \\
\hline 36 & 40123876477103981082575703570111021523 & \\
\hline 37 & 36821545755911193736497305295318821 & S21 \\
\hline 38 & 37922145892928094946547306395418821 & S27, S28 \\
\hline 39 & 3932313620098331012570213297102720122 & \\
\hline 40 & 35820695453851686535941277887017420 & S1, S18, S26, S29 (Gelfand-Tsetlin) \\
\hline 41 & 459285181111372015118112235614183436236 & \\
\hline 42 & 467291383221413315629116365831190537537 & \\
\hline 43 & 42325627083115961231387724200131625326 & \\
\hline 44 & 42525737108116261233387794201131625326 & S24 \\
\hline 45 & 39723636416103131075575363561110921523 & S23 \\
\hline 46 & 461287682251399315509115755814190337537 & \\
\hline 47 & 40023666377101751054673633480108921323 & \\
\hline 48 & 3932313620098331012570213297102720122 & \\
\hline 49 & 3932313620098331012570213297102720122 & \\
\hline & & \\
\hline
\end{tabular}




\begin{tabular}{|l|l|l|}
\hline Orbit & F-vector & Combinatorial equivalences \\
\hline 50 & 37922145892928094946547306395418821 & S2, S19 \\
\hline 51 & 42625997257120341298194204602147028629 & \\
\hline 52 & 42825947176117611251489474307135926327 & \\
\hline 53 & 41925226922112431184283733985124524025 & \\
\hline 54 & 466291783711428815879118705960194438037 & \\
\hline 55 & 443272976921286713982101974987158530430 & \\
\hline 56 & 453278778261301114021101224895153929329 & \\
\hline 57 & 469292683581418815679116635839190637537 & \\
\hline 58 & 458282579581328614398104725113162631331 & \\
\hline 59 & 472294984351433515854117965902192337737 & \\
\hline 60 & 440270476021268413752100144897156030130 & \\
\hline 61 & 472296785611472016525125266410214443243 & \\
\hline 62 & 457284280991372615153112665640184236336 & \\
\hline 63 & 465290282961409615588115945795188436836 & \\
\hline 64 & 459285181111372015118112235614183436236 & \\
\hline 65 & 42826087269120281294693774576146228529 & \\
\hline 66 & 44126817438122281305693694525143027628 & \\
\hline 67 & 41825106876111571175383213969124324025 & \\
\hline 68 & 40624426713109431158782453950124124025 & \\
\hline 69 & 373219959269474984968973267102420122 & \\
\hline 70 & 42725867144116811238388064209131725326 & \\
\hline 71 & 451278178401311114243103905089162331331 & \\
\hline 72 & 440270476021268413752100144897156030130 & \\
\hline 73 & 40624426713109431158782453950124124025 & \\
\hline 74 & 448276478001306114208103775087162331331 & \\
\hline 75 & 462287381811384615258113215656184436336 & \\
\hline 76 & 457284280991372615153112665640184236336 & \\
\hline 77 & 469292783641420315699116785845190737537 & \\
\hline 78 & 454280279031321614348104535110162631331 & \\
\hline 79 & 451278778791322114419105655200166732332 & \\
\hline 80 & 44127057584126111362298854823153729830 & \\
\hline 81 & 454280379141326314455105985231168733033 & \\
\hline 82 & 44126977532124651339196604685148528729 & \\
\hline 83 & 44527217593125501346196944694148628729 & \\
\hline 84 & 44126977532124651339196604685148528729 & \\
\hline 85 & 44527257617126111354697644728149528829 & \\
\hline 86 & 39723636416103131075575363561110921523 & \\
\hline 87 & 36821545755911193736497305295318821 & S5, S31 \\
\hline 88 & 452280179461338514654107715309169932732 & \\
\hline 89 & 43026247318120971297493294497141126927 & \\
\hline 90 & 456283480711367015083112105612183436236 & \\
\hline 91 & 43226337332121041297593414521143027628 & \\
\hline & & \\
\hline
\end{tabular}




\begin{tabular}{|l|l|l|}
\hline Orbit & -vector & Combinatorial equivalences \\
\hline 92 & 467291983591423015769117565892192237737 & \\
\hline 93 & 456283480711367015083112105612183436236 & \\
\hline 94 & 42625977244119981292693704575146228529 & \\
\hline 95 & 440270876301276913898101695001160331131 & \\
\hline 96 & 43226337332121041297593414521143027628 & \\
\hline 97 & 41224796810110831170783063967124324025 & \\
\hline 98 & 41525116945113911213386794174131325326 & \\
\hline 99 & 458284580921367615042111325543180035335 & \\
\hline 100 & 43726697447123191323695564642147528629 & \\
\hline 101 & 44127037569125621353197804746150228929 & \\
\hline 102 & 42725867144116811238388064209131725326 & \\
\hline 103 & 41925226922112431184283733985124524025 & \\
\hline 104 & 43726697447123191323695564642147528629 & \\
\hline 105 & 41124706776110121161782353933123423925 & \\
\hline 106 & 41324836808110431160681773871120123024 & \\
\hline 107 & 42525536988113171188883883987124524025 & \\
\hline 108 & 40524076518104421085175783571111021523 & \\
\hline 109 & 40524276638107511129679693785118122824 & $S 30$ \\
\hline 110 & 465290483121415215700117345907194038438 & \\
\hline 111 & 464290283231420415795118285960195638638 & \\
\hline 112 & 43826907559126081366799524868155230030 & \\
\hline 113 & 44527257617126111354697644728149528829 & \\
\hline 114 & 43726697447123191323695564642147528629 & \\
\hline 115 & 41124706776110121161782353933123423925 & \\
\hline 116 & 42425747139117371252989834332136726427 & \\
\hline 117 & 41925226922112431184283733985124524025 & \\
\hline 118 & 40123876477103981082575703570111021523 & \\
\hline 119 & 40524276638107511129679693785118122824 & S6 \\
\hline 120 & 464289382611401915483115035746186936636 & \\
\hline 121 & 454280679281328314448105435159164131531 & \\
\hline 122 & 451279479281337014676108405387174634234 & \\
\hline 123 & 444273677151291514053102735044161331231 & \\
\hline 124 & 466290983181413815644116505837190637537 & \\
\hline 125 & 456281579391327114398104805118162731331 & \\
\hline 126 & 42325617078115861230387674199131625326 & \\
\hline 127 & 42925807064114291197284023959122123224 & \\
\hline 128 & 43126267309120581291592904494142227528 & \\
\hline 129 & 42826027224118831268490874375137726527 & \\
\hline 130 & 443272776791283113927101474960157730330 & \\
\hline 131 & 43226377354121521302493564505141226927 & \\
\hline 132 & 451279379201334214620107705331171833433 & \\
\hline 133 & 43426327273118791255788834210130124625 & \\
\hline
\end{tabular}




\begin{tabular}{|l|l|l|}
\hline Orbit & F-vector & Combinatorial equivalences \\
\hline 134 & 452 2781 7813 13004 14042 10171 4944 1566 301 30 & \\
\hline 135 & 453280879691343314725108475366172733533 & \\
\hline 136 & 451279479281337014676108405387174634234 & \\
\hline 137 & 43326467390122361315094824589144827828 & \\
\hline 138 & 442271576291272713808100764948158730931 & \\
\hline 139 & 43226337332121041297593414521143027628 & \\
\hline 140 & 42325647096116321236888224227132425426 & \\
\hline 141 & 41324836808110431160681773871120123024 & \\
\hline 142 & 42725947196118271261490314347136926427 & \\
\hline 143 & 43126227281119731276991354390137926527 & \\
\hline 144 & 43126267309120581291592904494142227528 & \\
\hline 145 & 41024596725108811141180293802118322824 & \\
\hline 146 & 42825947176117611251489474307135926327 & \\
\hline 147 & 41925226922112431184283733985124524025 & \\
\hline 148 & 451278178401311114243103905089162331331 & \\
\hline 149 & 464290083101416815740117785933194838538 & \\
\hline 150 & 446275077571298514123103155058161531231 & \\
\hline 151 & 42025417021114961221887194184131425326 & \\
\hline 152 & 44127057584126111362298854823153729830 & \\
\hline 153 & 42525757119116511236387994208131725326 & \\
\hline 154 & 448276478011306714223103975102162931431 & \\
\hline 155 & 444273777241294914124103635115164732132 & \\
\hline 156 & 45227727753128301375598764750148628228 & \\
\hline 157 & 44227067565125291346096964684147328128 & \\
\hline 158 & 44127087602126551367699154821152529229 & \\
\hline 159 & 42725967207118501263390264324135025726 & \\
\hline 160 & 452278178131300414042101714944156630130 & \\
\hline 161 & 42725867144116811238388064209131725326 & \\
\hline 162 & 40023826467103881082075693570111021523 & \\
\hline 163 & 448276478001306114208103775087162331331 & \\
\hline 164 & 470294384441440516000119596011196738738 & \\
\hline 165 & 460285781171368414985110145430174033633 & \\
\hline 166 & 41825306996114661219887124183131425326 & \\
\hline 167 & 43426407325120251278891084348135325726 & \\
\hline 168 & 42525777132116871241888494235132525426 & \\
\hline 169 & 42525817160117721256490044339136826427 & \\
\hline 170 & 43026147255119281272491094382137826527 & \\
\hline 171 & 42225577075115971233388014220132325426 & \\
\hline 172 & 41124706772109881155681503863120023024 & S7 \\
\hline 173 & 42725867144116811238388064209131725326 & \\
\hline 174 & 40023826467103881082075693570111021523 & \\
\hline 175 & 464289882951411915649116735856191337637 & \\
\hline & & \\
\hline
\end{tabular}




\begin{tabular}{|l|l|l|}
\hline Orbit & F-vector & Combinatorial equivalences \\
\hline 176 & 442271876441275413822100564911156230130 & \\
\hline 177 & 44026987563125761358798644816153629830 & \\
\hline 178 & 42325627083115961231387724200131625326 & \\
\hline 179 & 452278178131300414042101714944156630130 & \\
\hline
\end{tabular}

Table 5: Orbits of maximal prime cones for $\mathscr{F} \ell_{5}$, the F-vectors of the corresponding polytopes, and combinatorially equivalent string polytopes resp. FFLV polytope.

\section{Algebraic invariants of the $\mathscr{F} \ell_{5}$ string polytopes}

The table below contains information on the $\mathscr{F} \ell_{5}$ string polytopes and the FFLV polytope for $\rho$. It shows the reduced expressions underlying the string polytopes, whether the polytopes satisfy the weak Minkowski property, the weight vectors constructed in $\$ 5$ and whether the corresponding initial ideal is prime. The last column contains information on unimodular equivalences among these polytopes. If there is no information in this column this means that there is no unimodular equivalence between this polytope and any other polytope in the table.

\begin{tabular}{|c|c|c|c|c|c|c|}
\hline & $\underline{w}_{0}$ & Normal & $\overline{M P}$ & Weight vector & Prime & Uni. Eq. \\
\hline S1 & 1213214321 & yes & yes & $\begin{array}{c}w_{1}=(0,512,384,112,0,256,96,768,608,480,0,64,320,832,15,14 \\
526,398,126,12,268,108,780,620,492,0,8,72,328,840)\end{array}$ & yes & $\begin{array}{l}\text { S18, S26, } \\
\text { S29 }\end{array}$ \\
\hline $\mathrm{S} 2$ & 1213243212 & yes & yes & $\begin{array}{c}w_{2}=(0,512,384,98,0,256,96,768,608,480,0,64,320,832,30,28,540, \\
412,123,24,280,120,792,632,504,0,16,80,336,848)\end{array}$ & yes & - \\
\hline S3 & 1213432312 & yes & no & $\begin{array}{c}w_{3}=(0,512,384,74,0,256,72,768,584,456,0,64,320,832,58,56,568, \\
440,111,48,304,108,816,620,492,0,32,96,352,864)\end{array}$ & no & - \\
\hline S4 & 1214321432 & yes & no & $\begin{array}{c}w_{4}=(0,512,384,56,0,256,48,768,560,432,0,32,288,800,120,112,624 \\
496,63,96,352,54,864,566,438,0,64,36,292,804)\end{array}$ & no & - \\
\hline S5 & 1232124321 & yes & yes & $\begin{array}{c}w_{5}=(0,512,288,224,0,256,192,768,704,432,0,128,384,896,15,14,526, \\
302,238,12,268,204,780,716,444,0,8,136,392,904)\end{array}$ & yes & - \\
\hline S6 & 1232143213 & yes & yes & $\begin{array}{c}w_{6}=(0,512,288,224,0,256,192,768,704,420,0,128,384,896,30,28,540, \\
316,252,24,280,216,792,728,437,0,16,144,400,912)\end{array}$ & yes & - \\
\hline S7 & 1232432123 & yes & yes & $\begin{array}{c}w_{7}=(0,512,260,196,0,256,192,768,704,390,0,128,384,896,60,56,568, \\
310,246,48,304,240,816,752,423,0,32,160,416,928)\end{array}$ & yes & - \\
\hline S8 & 1234321232 & yes & no & $\begin{array}{c}w_{8}=(0,512,264,152,0,256,144,768,656,396,0,128,384,896,120,112,624, \\
364,219,96,352,210,864,722,462,0,64,192,448,960)\end{array}$ & no & - \\
\hline S9 & 1234321323 & yes & no & $\begin{array}{c}w_{9}=(0,512,264,152,0,256,144,768,656,394,0,128,384,896,120,112,624, \\
362,222,96,352,212,864,724,459,0,64,192,448,960)\end{array}$ & no & - \\
\hline $\mathrm{S} 10$ & 1243212432 & yes & no & $\begin{array}{c}w_{10}=(0,512,272,112,0,256,96,768,608,344,0,64,320,832,240,224,736, \\
472,119,192,448,102,960,614,350,0,128,68,324,836)\end{array}$ & no & - \\
\hline
\end{tabular}




\begin{tabular}{|c|c|c|c|c|c|c|}
\hline & $\underline{w}_{0}$ & Normal & TMP & weight vector & Prime & Uni. Eq \\
\hline S11 & 1243214323 & yes & no & $\begin{array}{c}w_{11}=(0,512,272,112,0,256,96,768,608,338,0,64,320,832,240,224,736 \\
466,126,192,448,108,960,620,347,0,128,72,328,840)\end{array}$ & no & - \\
\hline $\mathrm{S} 12$ & 1321324321 & yes & no & $\begin{array}{c}w_{12}=(0,512,192,448,0,128,384,640,896,240,0,256,160,672,15,14,526 \\
206,462,12,140,396,652,908,252,0,8,264,168,680)\end{array}$ & no & - \\
\hline $\mathrm{S} 13$ & 1321343231 & yes & no & $\begin{array}{c}w_{13}=(0,512,192,448,0,128,384,640,896,228,0,256,160,672,29,28,540, \\
220,476,24,152,408,664,920,246,0,16,272,176,688)\end{array}$ & no & - \\
\hline $\mathrm{S} 14$ & 1321432143 & yes & no & $\begin{array}{c}w_{14}=(0,512,192,448,0,128,384,640,896,216,0,256,144,656,60,56,568, \\
248,504,48,176,432,688,944,219,0,32,288,146,658)\end{array}$ & no & - \\
\hline S15 & 1323432123 & yes & no & $\begin{array}{c}w_{15}=(0,512,132,388,0,128,384,640,896,198,0,256,192,704,60,56,568 \\
182,438,48,176,432,688,944,231,0,32,288,224,736)\end{array}$ & no & - \\
\hline S16 & 1324321243 & yes & no & $\begin{array}{c}w_{16}=(0,512,136,392,0,128,384,640,896,172,0,256,160,672,120,112,624, \\
236,492,96,224,480,736,992,175,0,64,320,162,674)\end{array}$ & no & - \\
\hline S17 & 1343231243 & yes & no & $\begin{array}{c}w_{17}=(0,512,48,304,0,32,288,544,800,60,0,256,40,552,240,224,736,188, \\
444,192,168,424,680,936,63,0,128,384,42,554)\end{array}$ & no & - \\
\hline S18 & 2123214321 & yes & yes & $\begin{array}{c}w_{18}=(0,256,768,112,0,512,96,384,352,864,0,64,576,448,15,14,270,782 \\
126,12,524,108,396,364,876,0,8,72,584,456)\end{array}$ & yes & $\begin{array}{l}\text { S1, S26 } \\
\text { S29 }\end{array}$ \\
\hline S19 & 2123243212 & yes & yes & $\begin{array}{c}w_{19}=(0,256,768,98,0,512,96,384,352,864,0,64,576,448,30,28,284,796 \\
123,24,536,120,408,376,888,0,16,80,592,464)\end{array}$ & yes & - \\
\hline S20 & 2123432132 & yes & no & $\begin{array}{c}w_{20}=(0,256,768,76,0,512,72,384,328,840,0,64,576,448,60,56,312,824, \\
111,48,560,106,432,362,874,0,32,96,608,480)\end{array}$ & no & - \\
\hline S21 & 2132134321 & yes & yes & $\begin{array}{c}w_{21}=(0,256,768,224,0,512,192,320,448,960,0,128,640,336,15,14,270 \\
782,238,12,524,204,332,460,972,0,8,136,648,344)\end{array}$ & yes & - \\
\hline $\mathrm{S} 22$ & 2132143214 & yes & yes & $\begin{array}{c}w_{22}=(0,256,768,224,0,512,192,320,448,960,0,128,640,328,30,28,284, \\
796,252,24,536,216,344,472,984,0,16,144,656,329)\end{array}$ & yes & - \\
\hline S23 & 2132343212 & yes & yes & $\begin{array}{c}w_{23}=(0,256,768,194,0,512,192,320,448,960,0,128,640,352,30,28,284, \\
796,219,24,536,216,344,472,984,0,16,144,656,368)\end{array}$ & yes & - \\
\hline S24 & 2132432124 & yes & yes & $\begin{array}{c}w_{24}=(0,256,768,196,0,512,192,320,448,960,0,128,640,336,60,56,312 \\
824,246,48,560,240,368,496,1008,0,32,160,672,337)\end{array}$ & yes & - \\
\hline S25 & 2134321324 & yes & no & $\begin{aligned} w_{25}= & (0,256,768,152,0,512,144,272,400,912,0,128,640,276,120,112 \\
& 368,880,222,96,608,212,340,468,980,0,64,192,704,277)\end{aligned}$ & no & - \\
\hline S26 & 2321234321 & yes & yes & $\begin{array}{c}w_{26}=(0,64,576,448,0,512,384,96,352,864,0,256,768,112,15,14,78, \\
590,462,12,524,396,108,364,876,0,8,264,776,120)\end{array}$ & yes & $\begin{array}{l}\mathrm{S} 1, \mathrm{~S} 18 \\
\mathrm{~S} 29\end{array}$ \\
\hline S27 & 2321243214 & yes & yes & $\begin{array}{c}w_{27}=(0,64,576,448,0,512,384,96,352,864,0,256,768,104,30,28,92, \\
604,476,24,536,408,120,376,888,0,16,272,784,105)\end{array}$ & yes & - \\
\hline S28 & 2321432134 & yes & yes & $\begin{array}{c}w_{28}=(0,64,576,448,0,512,384,72,328,840,0,256,768,74,60,56,120, \\
632,504,48,560,432,106,362,874,0,32,288,800,75)\end{array}$ & yes & - \\
\hline S29 & 2324321234 & yes & yes & $\begin{array}{c}w_{29}=(0,8,520,392,0,512,384,12,268,780,0,256,768,14,120,112,108, \\
620,492,96,608,480,78,334,846,0,64,320,832,15)\end{array}$ & yes & $\begin{array}{l}\text { S1, S18 } \\
\text { S26 }\end{array}$ \\
\hline S30 & 2343212324 & yes & yes & $\begin{array}{c}w_{30}=(0,16,528,304,0,512,288,24,280,792,0,256,768,28,240,224,216, \\
728,438,192,704,420,156,412,924,0,128,384,896,29)\end{array}$ & yes & - \\
\hline S31 & 2343213234 & yes & yes & $\begin{array}{c}w_{31}=(0,16,528,304,0,512,288,20,276,788,0,256,768,22,240,224,212, \\
724,444,192,704,424,150,406,918,0,128,384,896,23)\end{array}$ & yes & - \\
\hline FFLV & & yes & yes & $\begin{array}{l}w^{\text {reg }}=(0,4,6,6,0,3,4,6,6,9,0,2,4,6,4,3,4,7,8,2,3,5,4,6,8,0,1,2,3,4) \\
w^{\text {min }}=(0,3,4,3,0,2,2,4,3,5,0,1,2,3,1,1,1,3,3,1,1,2,1,2,3,0,1,1,1,1)\end{array}$ & \begin{tabular}{|l} 
yes \\
yes
\end{tabular} & \\
\hline
\end{tabular}

Table 6 String polytopes depending on $\underline{w}_{0}$ and the FFLV polytope for $\mathscr{F} \ell_{5}$ and $\rho$, their normality, the weak Minkowski property, the weight vectors constructed in $\$ 5$ primeness of the binomial initial ideals, and unimodular equivalences among the polytopes. 